\title{
Remarkably Facile Hexatriene Electrocyclizations as a Route to Functionalized Cyclohexenones via Ring Expansion of Cyclobutenones
}

\author{
Nabi A. Magomedov, Piero L. Ruggiero, and Yuchen Tang \\ Department of Chemistry, University of Rochester, Rochester, NY 14627-0216 \\ magomedov@chem.rochester.edu
}

\section{Supporting Information}

General Information. All reactions were carried out under an atmosphere of nitrogen in oven-dried glassware with magnetic stirring. Solvents and reagents were purified prior to use following the guidelines of Perrin and Armarego, ${ }^{1}$ except for THF, toluene, acetonitrile, dichloromethane and ether, which were dispensed using the Glass Contour solvent purification system. Air- and moisture-sensitive liquids and solutions were transferred via syringe or stainless steel cannula. Organic solutions were concentrated in vacuo below $45^{\circ} \mathrm{C}$ at approximately $20 \mathrm{~mm} \mathrm{Hg}$. Purification of reaction products was carried out by flash chromatography using EM Reagent silica gel 60 (230-400 mesh). Analytical thin layer chromatography was performed on EM Reagent $0.25 \mathrm{~mm}$ silica gel $60-F$ plates. Visualization was accomplished with UV light and potassium permanganate solution or ceric ammonium molybdate solution followed by heating. Melting points were measured with a Unimelt Thomas-Hoover melting point apparatus and are uncorrected. Infrared spectra were recorded on a Perkin Elmer 1600 series FT-IR spectrometer. ${ }^{1} \mathrm{H}$ NMR spectra were recorded on a Bruker Avance-400 (400 MHz) or Bruker AMX-400 (400 MHz) spectrometer and are reported in ppm using the solvent as an internal standard $\left(\mathrm{CDCl}_{3}\right.$ at $\left.7.26 \mathrm{ppm}\right)$. Data are reported as $(\mathrm{br}=$ broad, $\mathrm{s}=$ singlet, $\mathrm{d}$ $=$ doublet, $\mathrm{t}=$ triplet, $\mathrm{q}=$ quartet, $\mathrm{m}=$ multiplet; coupling constant(s) in Hz; integration). Proton-decoupled ${ }^{13} \mathrm{C}$ NMR spectra were recorded on a Bruker Avance-400 (100 MHz) or Bruker AMX-400 (100 MHz) spectrometer and are reported in ppm using the solvent as the internal standard $\left(\mathrm{CDCl}_{3}\right.$ at $\left.77.23 \mathrm{ppm}\right)$. High-resolution mass spectra were obtained on a VG-7070 spectrometer at the UC Riverside Mass Spectrometry Facility. 4Methyl-3-phenylcyclobutenone, ${ }^{2}$ 2,3-diethylcyclobutene-2-one, ${ }^{3}$ compound $\mathbf{1 1},{ }^{4}, 2,3$ dihydrothiophene-1,1-dioxide, ${ }^{5}$ (2,2-diphenyl)ethenesulfonylbenzene, ${ }^{6}$ and (hex-1Z-ene1 -sulfonyl)benzene ${ }^{7}$ were prepared according to the literature procedures. Except for the

\footnotetext{
${ }^{1}$ Armarego, W. L. F.; Perrin, D. D. Purification of Laboratory Chemicals; Butterworth-Heinemann: Bath, 2002.

${ }^{2}$ Schmit, C.; Sahraoui-Taleb, S.; Differding, E.; Dehasse-De Lombaert, C. G.; Ghosez, L. Tetrahedron Lett. 1984, 25, 5043-5046.

${ }^{3}$ Ammann, A. A.; Rey, M.; Dreiding, A. S. Helv. Chim. Acta 1987, 70, 321-328.

${ }^{4}$ Danheiser, R. L.; Savariar, S.; Cha, D. D. Org. Synth. 1990, 68, 32-40.

${ }^{5}$ Bailey, W. J.; Cummins, E. W. J. Am. Chem. Soc. 1954, 76, 1932.

${ }^{6}$ Ager, D. J. J. Chem. Soc., Perkin Trans. 1 1986, 183-194.

${ }^{7}$ Iwata, N.; Morioka, T.; Kobayashi, T.; Asada, T.; Kinoshita, H.; Inomata, K. Bull. Chem. Soc. Jpn. 1992, $65,1379-1388$.
} 
cyclopropyl substituted sulfone, all trans sulfones are known compounds and were prepared according to the following general procedure: ${ }^{8}$

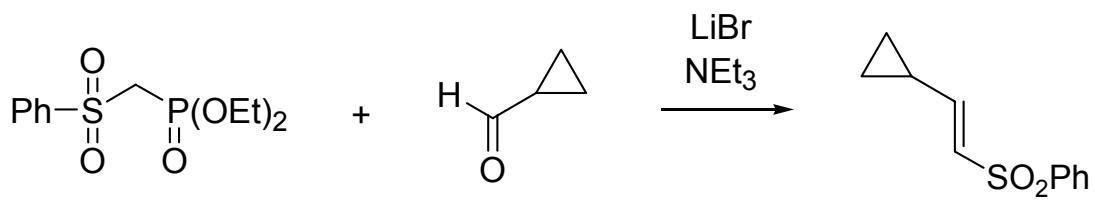

To a solution of $1.95 \mathrm{~g}(6.67 \mathrm{mmol})$ of (benzenesulfonyl)methylphosphonic acid diethyl ester and $0.695 \mathrm{~g}(8.01 \mathrm{mmol})$ of $\mathrm{LiBr}$ in $12 \mathrm{~mL}$ of $\mathrm{CH}_{3} \mathrm{CN}$ at room temperature, was added dropwise $1.02 \mathrm{~mL}(0.743 \mathrm{~g}, 7.34 \mathrm{mmol})$ of triethylamine. Once the reaction mixture became heterogenous, it was cooled to $0{ }^{\circ} \mathrm{C}$ and a solution of $0.50 \mathrm{~mL}(0.468 \mathrm{~g}$, $6.67 \mathrm{mmol}$ ) of cyclopropanecarboxaldehyde in $10 \mathrm{~mL}$ of $\mathrm{CH}_{3} \mathrm{CN}$ was added while vigorously stirring. After the addition was complete, the reaction mixture was warmed to room temperature and stirred for $4 \mathrm{~h}$. A solution of $30 \mathrm{~mL}$ of $0.1 \mathrm{~N} \mathrm{HCl}$ and $30 \mathrm{~mL}$ of $\mathrm{H}_{2} \mathrm{O}$ was added, and the reaction was diluted with $50 \mathrm{~mL} \mathrm{Et} \mathrm{t}_{2} \mathrm{O}$. The organic phase was separated and the aqueous one was extracted with $\mathrm{Et}_{2} \mathrm{O}(4 \times 40 \mathrm{~mL})$. The combined organic solutions were dried over $\mathrm{Na}_{2} \mathrm{SO}_{4}$, filtered, and concentrated in vacuo. Recrystallization from $\mathrm{Et}_{2} \mathrm{O} /$ hexanes provided $1.10 \mathrm{~g}$ of the desired sulfone as a colorless solid (79\% yield). Characterization data: $\mathrm{mp} 74-75{ }^{\circ} \mathrm{C}\left(\mathrm{Et}_{2} \mathrm{O} /\right.$ hexanes $)$; IR $\left(\mathrm{CHCl}_{3}\right) 3053$, $3007,2900,1625,1446,1306,1143,954 \mathrm{~cm}^{-1} ;{ }^{1} \mathrm{H}$ NMR $\left(400 \mathrm{MHz}, \mathrm{CDCl}_{3}\right) \delta$ 0.71-0.75 $(\mathrm{m}, 2 \mathrm{H}), 1.00-1.05(\mathrm{~m}, 2 \mathrm{H}), 1.53-1.61(\mathrm{~m}, 1 \mathrm{H}), 6.36(\mathrm{~d}, J=14.8 \mathrm{~Hz}, 1 \mathrm{H}), 6.44(\mathrm{dd}, J=$ $14.8,9.6 \mathrm{~Hz}, 1 \mathrm{H}), 7.54(\mathrm{t}, J=7.6 \mathrm{~Hz}, 1 \mathrm{H}), 7.54(\mathrm{t}, J=7.6 \mathrm{~Hz}, 2 \mathrm{H}), 7.88(\mathrm{dd}, J=7.6,1.4$ $\mathrm{Hz}, 2 \mathrm{H}) ;{ }^{13} \mathrm{C}$ NMR $\left(100 \mathrm{MHz}, \mathrm{CDCl}_{3}\right) \delta 151.9,141.0,132.9,129.1,127.2,126.7,45.9$, 13.8, 9.0; LRMS (APCI): Mass calcd for $\mathrm{C}_{11} \mathrm{H}_{13} \mathrm{O}_{2} \mathrm{~S}\left(\mathrm{M}+\mathrm{H}^{+}\right)$209.1. Found 209.0.

\section{Reactions of Cyclobutenones with Functionalized Vinyl Lithium Compounds}

General procedure A. To a solution of $200 \mathrm{mg}(0.77 \mathrm{mmol})$ of sulfone 9 in $5.7 \mathrm{~mL}$ of dry THF, cooled to $-50{ }^{\circ} \mathrm{C}$, was added $0.5 \mathrm{~mL}$ of a $1.87 \mathrm{M}$ solution of $n$-BuLi $(0.93$ mmol) in cyclohexane dropwise over 3 minutes. After 15 minutes at $-50{ }^{\circ} \mathrm{C}, 134 \mathrm{mg}$ (0.93 mmol) of 11 in $2 \mathrm{~mL}$ of dry THF was added dropwise. The reaction mixture was stirred at this temperature for 15 minutes and then warmed to room temperature over $1 \mathrm{~h}$, after which time TLC (silica gel, 3:1 hexanes/ethyl acetate) indicated complete consumption of starting sulfone. The reaction mixture was quenched with $5 \mathrm{~mL}$ of saturated aqueous $\mathrm{NH}_{4} \mathrm{Cl}$ solution. The aqueous phase was extracted with $\mathrm{CH}_{2} \mathrm{Cl}_{2}(3 \times 5$ $\mathrm{mL}$ ) and the combined organic solutions were dried over $\mathrm{Na}_{2} \mathrm{SO}_{4}$, filtered, and concentrated in vacuo. Flash chromatography (hexanes/ethyl acetate, 4:1) provided 252 mg of cyclohexenone $\mathbf{1 2}$ as a colorless solid ( $81 \%$ yield).

General procedure B. To a solution of $47 \mathrm{mg}(65 \mu \mathrm{L}, 0.47 \mathrm{mmol})$ of $i-\mathrm{Pr}_{2} \mathrm{NH}$ in $2.2 \mathrm{~mL}$ of dry THF cooled to $0{ }^{\circ} \mathrm{C}$, was added $0.25 \mathrm{~mL}$ of a $1.87 \mathrm{M}$ solution of $n$-BuLi $(0.47$ mmol) in cyclohexane, dropwise over 1 minute. After stirring for $15 \mathrm{~min}$ at $0{ }^{\circ} \mathrm{C}$, the solution was cooled to $-78{ }^{\circ} \mathrm{C}$ and a solution of $50 \mathrm{mg}(0.42 \mathrm{mmol})$ of $2,3-$ dihydrothiophene-1,1-dioxide in $0.5 \mathrm{~mL}$ of dry THF was added. The reaction was stirred for $15 \mathrm{~min}$ at $-78{ }^{\circ} \mathrm{C}$, and then a solution of $67 \mathrm{mg}(0.47 \mathrm{mmol})$ of cyclobutenone 11 in 1

\footnotetext{
${ }^{8}$ Adapted from Enders, D.; Berg, S.; Jandeleit, B. Org. Synth. 2000, 78, 177-188.
} 
$\mathrm{mL}$ of dry THF was added dropwise. The reaction mixture was stirred at $-78{ }^{\circ} \mathrm{C}$ for 20 min and then was warmed to room temperature over $1 \mathrm{~h}$, after which time TLC (silica gel, 2:1 hexanes/ethyl acetate) indicated complete consumption of the dihydrothiophene. The reaction mixture was quenched with $5 \mathrm{~mL}$ of saturated aqueous $\mathrm{NH}_{4} \mathrm{Cl}$. The aqueous phase was extracted with $\mathrm{CH}_{2} \mathrm{Cl}_{2}(3 \times 5 \mathrm{~mL})$ and the combined organic solutions were dried over $\mathrm{Na}_{2} \mathrm{SO}_{4}$, filtered, and concentrated in vacuo. Flash chromatography (hexanes/ethyl acetate, 1:1) provided $58 \mathrm{mg}$ of 1,1-dioxo-5-phenyl-1,2,3,3a,4,7ahexahydro- $1 \lambda^{6}$-benzo $[b]$ thiophen-7-one (52\% yield) as a white crystalline solid.

\section{Characterization data for all new compounds (in the order they appear in the text)}<smiles>O=C1C=C(c2ccccc2)C[C@H](c2ccccc2)[C@@H]1S(=O)(=O)[O-]</smiles>

3,5-Diphenyl-6-(toluene-4-sulfonyl)-cyclohex-2-enone (12). Following General procedure A, was obtained in $81 \%$ yield. Characterization data: colorless solid, mp 133-134 ${ }^{\circ} \mathrm{C}$ $\left(\mathrm{CH}_{2} \mathrm{Cl}_{2}\right.$ /Hexane); IR $\left(\mathrm{CHCl}_{3}\right) 3085,3060,3030,2918,2848,1658$, 1607, 1495, 1448, 1317, $1145 \mathrm{~cm}^{-1} ;{ }^{1} \mathrm{H}$ NMR (400 MHz, $\left.\mathrm{CDCl}_{3}\right) \delta 2.47(\mathrm{~s}, 3 \mathrm{H}), 3.16(\mathrm{~d}$, $J=19.0 \mathrm{~Hz}, 1 \mathrm{H}), 3.89(\mathrm{ddd}, J=19.0,6.4,2.5 \mathrm{~Hz}, 1 \mathrm{H}), 4.10(\mathrm{~s}, 1 \mathrm{H}), 4.64(\mathrm{~d}, J=6.4 \mathrm{~Hz}$, $1 \mathrm{H}), 6.63(\mathrm{~d}, J=2.5 \mathrm{~Hz}, 1 \mathrm{H}), 7.13-7.19(\mathrm{~m}, 2 \mathrm{H}), 7.20-7.30(\mathrm{~m}, 3 \mathrm{H}), 7.38(\mathrm{~d}, J=8.6 \mathrm{~Hz}$, $2 \mathrm{H}), 7.42-7.51(\mathrm{~m}, 3 \mathrm{H}), 7.58-7.62(\mathrm{~m}, 2 \mathrm{H}), 7.76(\mathrm{~d}, J=8.1 \mathrm{~Hz}, 2 \mathrm{H}) ;{ }^{13} \mathrm{C}$ NMR $(100$ $\left.\mathrm{MHz}, \mathrm{CDCl}_{3}\right) \delta 188.3,158.9,145.1,141.6,137.8,135.2,130.6,129.7,128.9,128.8$, 127.2, 127.1, 126.3, 125.0, 124.9, 73.9, 38.0, 31.3, 21.6; HRMS (DEI): Exact mass calcd for $\mathrm{C}_{25} \mathrm{H}_{23} \mathrm{O}_{3} \mathrm{~S}\left(\mathrm{M}+\mathrm{H}^{+}\right)$403.1368. Found 403.1359.<smiles>O=C1C=C(c2ccccc2)C[C@H](c2cccs2)[C@H]1S(=O)(=O)c1ccccc1</smiles>

6-Benzenesulfonyl-3-phenyl-5-thiophen-2-yl-cyclohex-2-enone (Entry 1). Following General procedure A, a colorless oil was obtained in $86 \%$ yield. Characterization data: IR $\left(\mathrm{CHCl}_{3}\right)$ 1657, 1601, $1307 \mathrm{~cm}^{-1} ;{ }^{1} \mathrm{H} \mathrm{NMR}\left(400 \mathrm{MHz}, \mathrm{CHCl}_{3}\right) \delta 3.20(\mathrm{~d}, J=18.6 \mathrm{~Hz}$, $1 \mathrm{H}), 3.89$ (ddd, $J=18.6,5.9,2.5 \mathrm{~Hz}, 1 \mathrm{H}), 4.14(\mathrm{~s}, 1 \mathrm{H}), 4.91(\mathrm{~d}, J=$ $5.9 \mathrm{~Hz}, 1 \mathrm{H}), 6.59(\mathrm{~d}, J=2.5 \mathrm{~Hz}, 1 \mathrm{H}), 6.83-6.85(\mathrm{~m}, 2 \mathrm{H}), 7.10(\mathrm{dd}, J=4.8,1.5 \mathrm{~Hz}, 1 \mathrm{H})$, 7.44-7.46 (m, 3H), 7.57-7.60 (m, 4H), 7.70 (t, $J=7.4$ Hz, 1H), 7.87 (dd, $J=8.6,1.4 \mathrm{~Hz}$, $2 \mathrm{H}) ;{ }^{13} \mathrm{C} \mathrm{NMR}\left(100 \mathrm{MHz}, \mathrm{CDCl}_{3}\right) \delta 187.9,158.9,144.5,138.5,138.2,134.5,131.0$, 129.4, 129.2, 129.1, 127.0, 126.7, 125.5, 125.2, 124.8, 73.8, 35.3, 33.2; HRMS (DEI): Exact mass calcd for $\mathrm{C}_{22} \mathrm{H}_{19} \mathrm{O}_{3} \mathrm{~S}_{2}\left(\mathrm{M}+\mathrm{H}^{+}\right)$395.0776. Found 395.0761.<smiles>CC(C)(C)[C@H]1CC(c2ccccc2)=CC(=O)C1S(=O)(=O)c1ccccc1</smiles>

6-Benzenesulfonyl-5-tert-butyl-3-phenyl-cyclohex-2-enone (Entry 2). Following General procedure $\mathbf{A}$, a colorless oil was obtained in 57\% yield. Characterization data: IR $\left(\mathrm{CHCl}_{3}\right)$ 2916, 1656, 1317, 1309, $1146 \mathrm{~cm}^{-1} ;{ }^{1} \mathrm{H}$ NMR $\left(400 \mathrm{MHz}, \mathrm{CDCl}_{3}\right) \delta 0.94(\mathrm{~s}, 9 \mathrm{H}), 2.97(\mathrm{~d}$, $J=19.5 \mathrm{~Hz}, 1 \mathrm{H}), 3.06(\mathrm{~d}, J=7.2 \mathrm{~Hz}, 1 \mathrm{H}), 3.45$ (ddd, $J=19.5,7.2,2.6 \mathrm{~Hz}, 1 \mathrm{H}), 4.08$ (s, $1 \mathrm{H}), 6.49(\mathrm{~d}, J=2.6 \mathrm{~Hz}, 1 \mathrm{H}), 7.45-7.47(\mathrm{~m}, 3 \mathrm{H}), 7.57-7.60(\mathrm{~m}, 4 \mathrm{H}), 7.70(\mathrm{t}, J=7.3 \mathrm{~Hz}$, $1 \mathrm{H}), 7.85(\mathrm{dd}, J=8.0,1.4 \mathrm{~Hz}, 2 \mathrm{H}) ;{ }^{13} \mathrm{C} \mathrm{NMR}\left(100 \mathrm{MHz}, \mathrm{CDCl}_{3}\right) \delta 189.2,160.7,138.2$, 138.0, 133.9, 130.5, 129.0, 128.9, 128.8, 126.2, 124.4, 70.8, 42.3, 34.8, 28.1, 26.7; HRMS (DEI): Exact mass calcd for $\mathrm{C}_{22} \mathrm{H}_{25} \mathrm{O}_{3} \mathrm{~S}\left(\mathrm{M}+\mathrm{H}^{+}\right)$369.1524. Found 369.1531.<smiles>CCC1=C(CC)C(=O)[C@H](S(=O)(=O)O[Na])[C@H](c2ccccc2)C1</smiles> 
yield. Characterization data: thick colorless oil, IR $\left(\mathrm{CHCl}_{3}\right) 2968,2934,1655,1119 \mathrm{~cm}^{-}$ ${ }^{1} ;{ }^{1} \mathrm{H}$ NMR $\left(400 \mathrm{MHz}, \mathrm{CDCl}_{3}\right) \delta 0.99(\mathrm{t}, J=7.2 \mathrm{~Hz}, 3 \mathrm{H}) ; 1.11(\mathrm{t}, J=7.3 \mathrm{~Hz}, 3 \mathrm{H}), 2.28-$ $2.47(\mathrm{~m}, 4 \mathrm{H}), 2.47(\mathrm{~s}, 3 \mathrm{H}), 2.62(\mathrm{~d}, J=19.0 \mathrm{~Hz}, 1 \mathrm{H}), 3.49(\mathrm{dd}, J=19.0,6.5 \mathrm{~Hz}, 1 \mathrm{H})$, $4.02(\mathrm{~s}, 1 \mathrm{H}), 4.39(\mathrm{~d}, J=6.5 \mathrm{~Hz}, 1 \mathrm{H}), 7.11(\mathrm{~d}, J=7.1 \mathrm{~Hz}, 2 \mathrm{H}), 7.20-7.28(\mathrm{~m}, 3 \mathrm{H}), 7.36$ $(\mathrm{d}, J=8.2 \mathrm{~Hz}, 2 \mathrm{H}), 7.71(\mathrm{~d}, J=8.2 \mathrm{~Hz}, 2 \mathrm{H}) ;{ }^{13} \mathrm{C} \mathrm{NMR}\left(100 \mathrm{MHz}, \mathrm{CDCl}_{3}\right) \delta 188.0$, 159.5, 145.0, 142.3, 136.9, 135.5, 129.6, 128.8, 128.7, 127.1, 74.0, 37.3, 33.5, 27.9, 21.6, 18.5, 13.5, 11.9; HRMS (EI): Exact mass calcd for $\mathrm{C}_{23} \mathrm{H}_{26} \mathrm{O}_{3} \mathrm{~S}\left(\mathrm{M}^{+}\right)$382.1597. Found 382.1600 .<smiles></smiles>

6-Benzenesulfonyl-2,3-diethyl-5,5-diphenyl-cyclohex-2-enone (Entry 4). Following General procedure A, was obtained in 63\% yield. Characterization data: white crystalline solid, $\mathrm{mp} 174-176{ }^{\circ} \mathrm{C}$ $\left(\mathrm{CH}_{2} \mathrm{Cl}_{2}\right.$ /hexane); IR $\left(\mathrm{CHCl}_{3}\right)$ 3058, 2970, 2933, 1660, 1625, 1447, $1318,1148 \mathrm{~cm}^{-1} ;{ }^{1} \mathrm{H}$ NMR $\left(400 \mathrm{MHz}, \mathrm{CDCl}_{3}\right) \delta 0.82(\mathrm{t}, J=7.5 \mathrm{~Hz}$, $3 \mathrm{H}), 0.97(\mathrm{t}, J=7.6 \mathrm{~Hz}, 3 \mathrm{H}), 2.15-2.39(\mathrm{~m}, 4 \mathrm{H}), 3.21(\mathrm{~d}, J=17.9 \mathrm{~Hz}, 1 \mathrm{H}), 4.50(\mathrm{~d}, J=$ $17.9 \mathrm{~Hz}, 1 \mathrm{H}), 5.03(\mathrm{~d}, J=1.8 \mathrm{~Hz}, 1 \mathrm{H}), 7.05-7.20(\mathrm{~m}, 10 \mathrm{H}), 7.35-7.39(\mathrm{~m}, 2 \mathrm{H}), 7.46-7.54$ (m, 3H); ${ }^{13} \mathrm{C}$ NMR $\left(100 \mathrm{MHz}, \mathrm{CDCl}_{3}\right) \delta 189.5,159.2,146.2,143.5,140.9,136.6,133.2$, $128.7,128.3,128.2,128.1,127.0,126.6,126.3,126.0,78.4,49.8,37.7,28.0,18.5,13.3$, 11.5; HRMS (DEI): Exact mass calcd for $\mathrm{C}_{28} \mathrm{H}_{29} \mathrm{O}_{3} \mathrm{~S}\left(\mathrm{M}+\mathrm{H}^{+}\right)$445.1832. Found 445.1836 .<smiles>CC(C)=CC([R5](=O)c1ccccc1)C(=O)C=C(C)c1ccccc1</smiles>

\section{5-Benzenesulfonyl-7-methyl-2-phenyl-octa-2,6-dien-4-one}

(16). Following General procedure A, a thick colorless oil was obtained in $85 \%$ yield. Characterization data: IR $\left(\mathrm{CHCl}_{3}\right) 1679,1593,1446$, $1307 \mathrm{~cm}^{-1} ;{ }^{1} \mathrm{H}$ NMR $\left(400 \mathrm{MHz}, \mathrm{CDCl}_{3}\right) \delta 1.51(\mathrm{~d}, J=1.0 \mathrm{~Hz}, 3 \mathrm{H})$, $1.80(\mathrm{~d}, J=1.0 \mathrm{~Hz}, 3 \mathrm{H}), 2.55(\mathrm{~d}, J=1.0 \mathrm{~Hz}, 3 \mathrm{H}), 4.92(\mathrm{~d}, J=10.3$ $\mathrm{Hz}, 1 \mathrm{H}), 5.49(\mathrm{dm}, J=10.3 \mathrm{~Hz}, 1 \mathrm{H}), 6.84(\mathrm{~d}, J=1.0 \mathrm{~Hz}, 1 \mathrm{H}), 7.42-7.44(\mathrm{~m}, 3 \mathrm{H}), 7.53-$ $7.58(\mathrm{~m}, 4 \mathrm{H}), 7.65(\mathrm{t}, J=6.4 \mathrm{~Hz}, 1 \mathrm{H}), 7.87(\mathrm{~d}, J=7.2 \mathrm{~Hz}, 2 \mathrm{H}) ;{ }^{13} \mathrm{C}$ NMR $(100 \mathrm{MHz}$, $\left.\mathrm{CDCl}_{3}\right) \delta 189.2,158.3,143.7,141.9,137.9,133.8,129.7,129.2,128.8,128.6,126.7$, 123.2, 112.3, 76.9, 26.1, 19.0, 18.4; HRMS (DEI): Exact mass calcd for $\mathrm{C}_{21} \mathrm{H}_{23} \mathrm{O}_{3} \mathrm{~S}$ $\left(\mathrm{M}+\mathrm{H}^{+}\right)$355.1368. Found 355.1385.

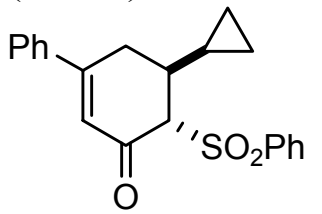

6-Benzenesulfonyl-5-cyclopropyl-3-phenylcyclohex-2-enone (Entry 5). Following General procedure A, was obtained in $80 \%$ yield. Characterization data: colorless solid, $\mathrm{mp} 110-111^{\circ} \mathrm{C}$ (Ethyl Acetate/Hexane); IR $\left(\mathrm{CHCl}_{3}\right)$ 3063, 3001, 2924, 2851, 1659, 1652, $1606,1447,1309,1251,1149 \mathrm{~cm}^{-1} ;{ }^{1} \mathrm{H}$ NMR $\left(400 \mathrm{MHz}, \mathrm{CDCl}_{3}\right) \delta$ 0.21-0.27 (m, 1H), 0.34-0.41 (m, 1H), 0.49-0.61 (m, 2H), 0.93-1.03 (m, 1H), 2.32 (dd, $J$ $=10.7,5.7 \mathrm{~Hz}, 1 \mathrm{H}), 2.94(\mathrm{~d}, J=18.5 \mathrm{~Hz}, 1 \mathrm{H}), 3.61(\mathrm{ddd}, J=18.5,5.7,2.5 \mathrm{~Hz}, 1 \mathrm{H}), 3.96$ (s, 1H), $6.54(\mathrm{~d}, J=2.5 \mathrm{~Hz}, 1 \mathrm{H}), 7.45-7.46(\mathrm{~m}, 3 \mathrm{H}), 7.59(\mathrm{~m}, 4 \mathrm{H}), 7.68(\mathrm{t}, J=7.2 \mathrm{~Hz}$, $1 \mathrm{H}), 7.84(\mathrm{~d}, J=7.5 \mathrm{~Hz}, 2 \mathrm{H}) ;{ }^{13} \mathrm{C}$ NMR $\left(100 \mathrm{MHz}, \mathrm{CDCl}_{3}\right) \delta 189.0,159.5,138.6,138.5$, 134.3, 130.8, 129.3, 129.1, 126.6, 124.1, 73.8, 40.0, 31.5, 16.0, 6.3, 5.0; HRMS (DEI) Exact mass calcd for $\mathrm{C}_{21} \mathrm{H}_{21} \mathrm{O}_{3} \mathrm{~S}\left(\mathrm{M}+\mathrm{H}^{+}\right)$353.1211. Found 353.1205.

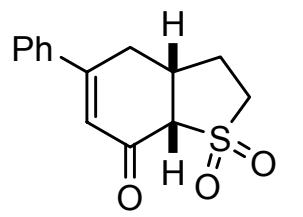

1,1-Dioxo-5-phenyl-1,2,3,3a,4,7a-hexahydro-1 $\lambda^{6}$-benzo[b]thiophen7-one (Entry 6). Following General procedure B, was obtained in $52 \%$ yield. Characterization data: colorless solid, mp $163-164{ }^{\circ} \mathrm{C}$ 
(Ether/ $\left.\mathrm{CH}_{2} \mathrm{Cl}_{2} / \mathrm{Hexane}\right)$; IR $\left(\mathrm{CHCl}_{3}\right)$ 3059, 3005, 2950, 2850, 1656, 1604, 1446, 1308, 1140, $1117 \mathrm{~cm}^{-1} ;{ }^{1} \mathrm{H}$ NMR (400 MHz, $\left.\mathrm{CDCl}_{3}\right) \delta$ 2.27-2.35 (m, 1H), 2.40-2.49 (m, $\left.1 \mathrm{H}\right)$, 2.90-3.03 (m, 2H), 3.27-3.36 (m, 3H), $3.96(\mathrm{~d}, J=7.6 \mathrm{~Hz}, 1 \mathrm{H}), 6.60(\mathrm{~s}, 1 \mathrm{H}), 7.41-7.47$ $(\mathrm{m}, 3 \mathrm{H}), 7.52-7.55(\mathrm{~m}, 2 \mathrm{H}) ;{ }^{13} \mathrm{C}$ NMR $\left(100 \mathrm{MHz}, \mathrm{CDCl}_{3}\right) \delta 186.6,159.0,138.0,131.1$, 129.2, 126.6, 125.2, 67.8, 51.0, 35.8, 29.3, 26.4; HRMS (DEI): Exact mass calcd for $\mathrm{C}_{14} \mathrm{H}_{15} \mathrm{O}_{3} \mathrm{~S}\left(\mathrm{M}+\mathrm{H}^{+}\right)$263.0742. Found 263.0738.

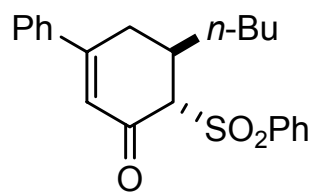

6-Benzenesulfonyl-5-butyl-3-phenylcyclohex-2-enone (Entry 7). $72 \%$ yield. Characterization data: thick colorless oil; IR $\left(\mathrm{CH}_{2} \mathrm{Cl}_{2}\right)$ 3057, 2932, 1657, 1607, 1448, 1319, $1150 \mathrm{~cm}^{-1}$; ${ }^{1} \mathrm{H}$ NMR $(400 \mathrm{MHz}$, $\left.\mathrm{CDCl}_{3}\right) \delta 0.86(\mathrm{t}, J=7.0 \mathrm{~Hz}, 3 \mathrm{H}), 1.25-1.34(\mathrm{~m}, 3 \mathrm{H}), 1.42-1.52(\mathrm{~m}$, $3 \mathrm{H}), 2.74(\mathrm{~d}, J=18.7 \mathrm{~Hz}, 1 \mathrm{H}), 3.24(\mathrm{~m}, 1 \mathrm{H}), 3.60(\mathrm{ddd}, J=18.7,5.6,2.5 \mathrm{~Hz}, 1 \mathrm{H}), 3.77$ $(\mathrm{s}, 1 \mathrm{H}), 6.49(\mathrm{~d}, J=2.5 \mathrm{~Hz}, 1 \mathrm{H}), 7.42-7.45(\mathrm{~m}, 3 \mathrm{H}), 7.54-7.59(\mathrm{~m}, 4 \mathrm{H}), 7.67$ (t, $J=7.3$ $\mathrm{Hz}, 1 \mathrm{H}), 7.84(\mathrm{~d}, J=7.5 \mathrm{~Hz}, 2 \mathrm{H}) ;{ }^{13} \mathrm{C} \mathrm{NMR}\left(100 \mathrm{MHz}, \mathrm{CDCl}_{3}\right) \delta 14.1,22.6,29.6,30.7$, 33.5, 33.6, 73.6, 124.0, 126.6, 129.1, 129.1, 129.3, 130.9, 134.3, 138.6, 138.8, 159.3, 189.1; HRMS (DEI): Exact mass calcd for $\mathrm{C}_{22} \mathrm{H}_{25} \mathrm{O}_{3} \mathrm{~S}\left(\mathrm{M}+\mathrm{H}^{+}\right)$369.1524. Found 369.1534 .

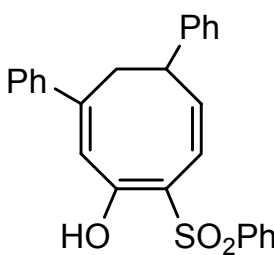

2-Benzenesulfonyl-5,7-diphenylcycloocta-1,3,7-trienol (21). Following General procedure A, was obtained in $45 \%$ yield. Characterization data: white crystalline solid, mp $155-156{ }^{\circ} \mathrm{C} \quad\left(\mathrm{CH}_{2} \mathrm{Cl}_{2} /\right.$ hexane), IR $\left(\mathrm{CHCl}_{3}\right)$ 3185, 3060, 1587, 1446, 1297, $1221 \mathrm{~cm}^{-1}$; ${ }^{1} \mathrm{H} \mathrm{NMR}(400 \mathrm{MHz}$, $3.41(\mathrm{~m}, 1 \mathrm{H}), 5.73(\mathrm{dd}, J=12.4,5.6 \mathrm{~Hz}, 1 \mathrm{H}), 6.14(\mathrm{~d}, J=12.0 \mathrm{~Hz}, 1 \mathrm{H}), 6.40(\mathrm{~s}, 1 \mathrm{H})$, $6.49(\mathrm{~d}, J=7.6 \mathrm{~Hz}, 2 \mathrm{H}), 7.05-7.15(\mathrm{~m}, 3 \mathrm{H}), 7.39-7.40(\mathrm{~m}, 3 \mathrm{H}), 7.47-7.48(\mathrm{~m}, 2 \mathrm{H}), 7.63$ $(\mathrm{dd}, J=8.0,8.0 \mathrm{~Hz}, 2 \mathrm{H}), 7.75(\mathrm{t}, J=7.2 \mathrm{~Hz}, 1 \mathrm{H}), 8.03$ (d, $J=8.0 \mathrm{~Hz}, 2 \mathrm{H}), 11.01(\mathrm{~s}, 1 \mathrm{H})$; ${ }^{13} \mathrm{C}$ NMR $\left(100 \mathrm{MHz}, \mathrm{CDCl}_{3}\right) \delta 160.6,150.3,143.0,141.4,139.9$ (bs), 137.0 (bs), 133.8, 129.6, 129.2, 129.0, 128.7, 127.8, 127.3, 126.8, 126.7, 121.9, 118.8, 107.8, 43.5, 37.8; HRMS (DEI): Exact mass calcd for $\mathrm{C}_{26} \mathrm{H}_{23} \mathrm{O}_{3} \mathrm{~S}\left(\mathrm{M}+\mathrm{H}^{+}\right)$415.1368. Found 415.1355.<smiles>CN1CCC(c2ccccc2)=C(Br)C1=O</smiles>

3-Bromo-1-methyl-4-phenyl-5,6-dihydro-1H-pyridin-2-one (22). To a solution of $1.06 \mathrm{~g}$ (5.67 mmol) of 1-methyl-4-phenyl-5,6-dihydro-1Hpyridin-2-one ${ }^{9}$ in $10 \mathrm{~mL}$ of $\mathrm{CH}_{2} \mathrm{Cl}_{2}$, cooled to $0{ }^{\circ} \mathrm{C}$ (water/ice bath), was added $337 \mu \mathrm{L}(6.52 \mathrm{mmol})$ of $\mathrm{Br}_{2}$. The reaction mixture was stirred at room temperature for $10 \mathrm{~min}$ and then concentrated in vacuo to give orange foam. The foam was dissolved in $5 \mathrm{~mL}$ of dioxane and $1.72 \mathrm{~g}$ of DBU (11.34 mmol) was added in one portion. After $1 \mathrm{~h}$ at room temperature, TLC (silica gel, EtOAc) indicated complete consumption of starting material. The reaction mixture was partitioned between $30 \mathrm{~mL}$ of $1 \mathrm{~N}$ aqueous $\mathrm{HCl}$ and $150 \mathrm{~mL}$ of $\mathrm{CH}_{2} \mathrm{Cl}_{2}$. The organic solution was dried $\left(\mathrm{MgSO}_{4}\right)$, concentrated in vacuo to give a solid residue. The residue was flash chromatographed over $20 \mathrm{~g}$ of silica gel (EtOAc:Hex, 3:1) to produce $1.11 \mathrm{~g}$ (74\%) of amide 22 as a white crystalline solid: mp 116-117 ${ }^{\circ} \mathrm{C}$; IR $\left(\mathrm{CH}_{2} \mathrm{Cl}_{2}\right) 3054,1652$, 1490, 1340, 1270, 1264, $1258 \mathrm{~cm}^{-1} ;{ }^{1} \mathrm{H}$ NMR (400 MHz, $\left.\mathrm{CDCl}_{3}\right) \delta 2.79(\mathrm{t}, J=7.0 \mathrm{~Hz}$,

\footnotetext{
${ }^{9}$ Soldatenkov, A. T.; Temesgen, A. V.; Bekro, I. A.; Soldatova, S. A.; Golovtsov, N. I.; Sergeeva, N. D. Chem. Heterocycl. Compd. 2000, 36, 1426-1430.
} 
$2 \mathrm{H}), 3.12(\mathrm{~s}, 3 \mathrm{H}), 3.55(\mathrm{t}, J=7.0 \mathrm{~Hz}, 2 \mathrm{H}), 7.35-7.43(\mathrm{~m}, 5 \mathrm{H}) ;{ }^{13} \mathrm{C}$ NMR $(100 \mathrm{MHz}$, $\left.\mathrm{CDCl}_{3}\right) \delta 32.3,36.0,47.1,116.7,127.5,128.5,128.8,140.0,149.3,161.2 ;$ LRMS (APCI): Mass calcd for $\mathrm{C}_{12} \mathrm{H}_{13} \mathrm{BrNO}\left(\mathrm{M}+\mathrm{H}^{+}\right) 266.0$ and 268.0. Found 266.0 and 268.0 (1:1 in intensity).<smiles>CN1CCC2(c3ccccc3)CC(c3ccccc3)=CC(O)=C2C1=O</smiles>

8-Hydroxy-2-methyl-4a,6-diphenyl-3,4,4a,5-tetrahydro-2Hisoquinolin-1-one (24). To a solution of $58 \mathrm{mg}(0.218 \mathrm{mmol})$ of bromide 22 in $1 \mathrm{~mL}$ of $\mathrm{THF}$, cooled to $-92{ }^{\circ} \mathrm{C}\left(\mathrm{CH}_{2} \mathrm{Cl}_{2} /\right.$ liquid $\left.\mathrm{N}_{2}\right)$ was added $120 \mu \mathrm{L}$ of $2 \mathrm{M}$ solution of $n$-BuLi in cyclohexane $(0.240$ mmol) dropwise over a period of 1 minute. After 5 minutes at bath temperature, a solution of $31 \mathrm{mg}(0.218 \mathrm{mmol})$ cyclobutenone in $0.5 \mathrm{~mL}$ of THF was added dropwise via syringe over a period of 3 minutes. The reaction mixture was left to warm to room temperature and then heated in an oil bath at $65{ }^{\circ} \mathrm{C}$ for $1 \mathrm{~h}$. The reaction mixture was cooled down to room temperature and then quenched with $1 \mathrm{~mL}$ of saturated aqueous $\mathrm{NH}_{4} \mathrm{Cl}$ solution. The mixture was partitioned between $100 \mathrm{~mL}$ of dichloromethane and $10 \mathrm{~mL}$ of water. The organic phase was separated and the aqueous one was extracted with dichloromethane $(20 \mathrm{~mL})$. The combined organic solutions were dried $\left(\mathrm{MgSO}_{4}\right)$, and then concentrated in vacuo to provide thick yellow oil. The oil was flash chromatographed over $15 \mathrm{~g}$ of silica gel (EtOAc:Hex, 1:1, then 2:1) to provide 37 $\mathrm{mg}(52 \%)$ of the bicyclic amide 24 . Characterization data for 24: yellow crystalline solid, mp 167-168 ${ }^{\circ} \mathrm{C}\left(\mathrm{CH}_{2} \mathrm{Cl}_{2} / \mathrm{Hexane}\right)$; IR $\left(\mathrm{CH}_{2} \mathrm{Cl}_{2}\right)$ 3070, 2925, 1643, 1586, 1503, 1446, $1368,1323 \mathrm{~cm}^{-1} ;{ }^{1} \mathrm{H}$ NMR $\left(400 \mathrm{MHz}, \mathrm{CDCl}_{3}\right) \delta 2.26(\mathrm{ddd}, J=12.9,12.9,4.7 \mathrm{~Hz}, 1 \mathrm{H})$, 2.38 (ddd, $J=12.9,3.4,2.0 \mathrm{~Hz}, 1 \mathrm{H}), 2.89$ (ddd, $J=12.9,12.5,3.4 \mathrm{~Hz}, 1 \mathrm{H}), 2.95$ (dd, $J=$ 16.4, $2.9 \mathrm{~Hz}, 1 \mathrm{H}), 2.97$ (s, 3H), 3.09 (ddd, $J=12.5,4.7,2.0 \mathrm{~Hz}, 1 \mathrm{H}), 3.27$ (d, $J=16.4$ $\mathrm{Hz}, 1 \mathrm{H}), 6.29(\mathrm{~d}, J=2.9 \mathrm{~Hz}, 1 \mathrm{H}), 7.21(\mathrm{t}, J=7.1 \mathrm{~Hz}, 1 \mathrm{H}), 7.26-7.32(\mathrm{~m}, 7 \mathrm{H}), 7.37-7.38$ $(\mathrm{m}, 2 \mathrm{H}), 14.56(\mathrm{bs}, 1 \mathrm{H}) ;{ }^{13} \mathrm{C} \mathrm{NMR}\left(100 \mathrm{MHz}, \mathrm{CDCl}_{3}\right) \delta 34.3,37.2,41.9,42.4,46.4,97.4$, 120.7, 125.9, 126.9, 127.0, 128.6, 128.7, 128.8, 139.2, 143.9, 145.3, 165.6, 169.7; HRMS (ESI): Exact mass calcd for $\mathrm{C}_{22} \mathrm{H}_{22} \mathrm{NO}_{2}\left(\mathrm{M}+\mathrm{H}^{+}\right)$332.1645. Found 332.1650.

\section{Control Experiments}

a) Reaction of 11 with $\beta$-(E)-lithiostyrene.

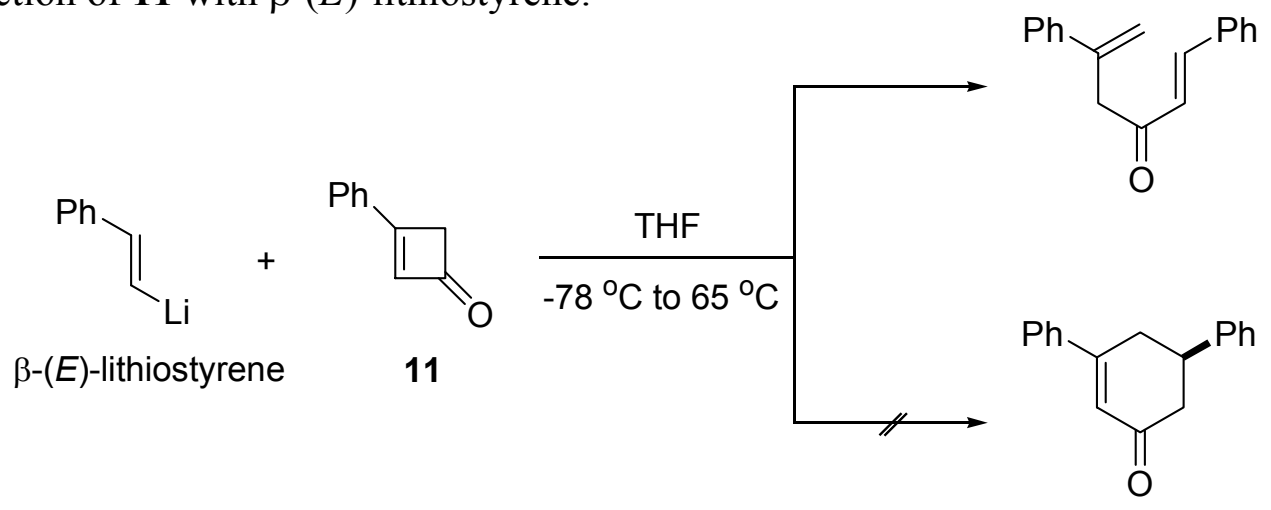

To a solution of $45 \mu \mathrm{L}$ of $\beta$ - $(E)$-bromostyrene $(63 \mathrm{mg}, 0.35 \mathrm{mmol})$ in $2 \mathrm{~mL}$ of dry THF cooled to $-78{ }^{\circ} \mathrm{C}$, was added dropwise $0.54 \mathrm{~mL}$ of a $1.30 \mathrm{M}$ solution of $t$-BuLi in 
pentane $(0.70 \mathrm{mmol})$. After stirring 15 minutes at $-78{ }^{\circ} \mathrm{C}, 50 \mathrm{mg}(0.35 \mathrm{mmol})$ of $\mathbf{1 1}$ in $1.5 \mathrm{~mL}$ of dry THF was added over a period of 1 minute. The reaction mixture was stirred at bath temperature for 20 minutes then at room temperature for 2 hours. Then the reaction mixture was quenched with $1 \mathrm{~mL}$ of saturated aqueous $\mathrm{NH}_{4} \mathrm{Cl}$. The aqueous phase was extracted with $\mathrm{CH}_{2} \mathrm{Cl}_{2}(3 \times 5 \mathrm{~mL})$ and the combined organic solutions were dried over $\mathrm{Na}_{2} \mathrm{SO}_{4}$, filtered, and concentrated in vacuo. Flash chromatography (hexanes/ethyl acetate, 4:1) provided $38 \mathrm{mg}$ (44\%) of 1,5-diphenylhexa-1,5-dien-3-one as a yellow oil. Characterization data: IR $\left(\mathrm{CHCl}_{3}\right)$ 3081, 3057, 3027, 2918, 1684, 1610, 1576, 1496, $979 \mathrm{~cm}^{-1} ;{ }^{1} \mathrm{H}$ NMR (400 MHz, $\left.\mathrm{CDCl}_{3}\right) \delta 3.83(\mathrm{~s}, 2 \mathrm{H}), 5.27(\mathrm{~s}, 1 \mathrm{H}), 5.65(\mathrm{~s}$, $1 \mathrm{H}), 6.87(\mathrm{~d}, J=16.0 \mathrm{~Hz}, 1 \mathrm{H}), 7.34(\mathrm{~m}, 6 \mathrm{H}), 7.44(\mathrm{~m}, 2 \mathrm{H}), 7.51(\mathrm{~m}, 2 \mathrm{H}), 7.62(\mathrm{~d}, J=$ 16.0 Hz, 1H); ${ }^{13} \mathrm{C}$ NMR $\left(100 \mathrm{MHz}, \mathrm{CDCl}_{3}\right) \delta 197.4,143.2,141.7,140.0,134.4,130.5$, 128.9, 128.5, 128.4, 127.9, 125.9, 124.7, 116.6, 48.5; LRMS (APCI): Mass calcd for $\mathrm{C}_{18} \mathrm{H}_{17} \mathrm{O}\left(\mathrm{M}+\mathrm{H}^{+}\right)$249.1. Found 249.1.

In an attempt to thermally induce the $6 \pi$-ERC, the same experiment was repeated and the reaction mixture was heated under reflux. Analysis of an aliquot collected after 1 $\mathrm{h}$ at $65{ }^{\circ} \mathrm{C}$ indicated that the reaction mixture still contained only the ring opened enone. After $14 \mathrm{~h}$ at reflux, this reaction produced a complex mixture of products. Careful analysis of the crude ${ }^{1} \mathrm{H}$ NMR of the reaction mixture revealed no peaks characteristic of 3,5-diphenylcyclohex-2-enone. ${ }^{10}$

b) Reaction of 10 and 11 at $-78{ }^{\circ} \mathrm{C}$.

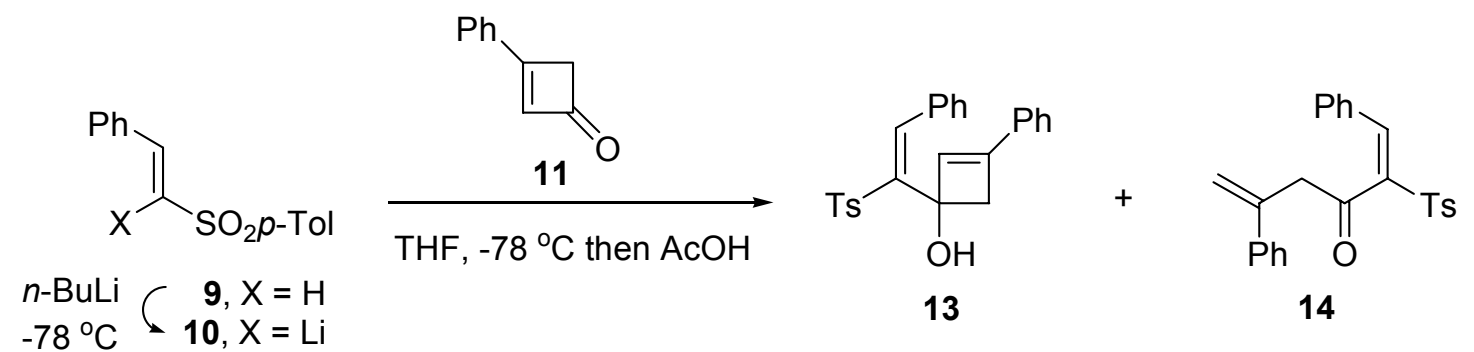

To a solution of $85 \mathrm{mg}(0.33 \mathrm{mmol})$ of sulfone 9 in $1 \mathrm{~mL}$ of THF, cooled to -78 ${ }^{\circ} \mathrm{C}$, was added $180 \mu \mathrm{L}(0.36 \mathrm{mmol})$ of $2 \mathrm{M}$ solution of $n$-BuLi in cyclohexane. After 10 min at bath temperature, a solution of $43 \mathrm{mg}(0.30 \mathrm{mmol})$ of $11 \mathrm{in} 0.5 \mathrm{~mL}$ of THF was added dropwise via syringe. The reaction mixture was stirred at bath temperature for $1 \mathrm{~h}$ and then quenched with $36 \mathrm{mg}(0.60 \mathrm{mmol})$ of glacial acetic acid. The reaction mixture was partitioned between $5 \mathrm{~mL}$ of water and $30 \mathrm{~mL}$ of $\mathrm{CH}_{2} \mathrm{Cl}_{2}$, the organic layer was separated, dried $\left(\mathrm{MgSO}_{4}\right)$, and concentrated in vacuo to provide a thick oil. The oil was was flash chromatographed over $15 \mathrm{~g}$ of silica gel (EtOAc:Hex, 1:3) to afford $74 \mathrm{mg}$ (61\%) of $\mathbf{1 3}$ and $28 \mathrm{mg}(23 \%)$ of $\mathbf{1 4}$ as thick colorless oils: Characterization data for 13: IR (neat) 3492 (br), 1658, 1596, 1447, 1311, 1301, $1144 \mathrm{~cm}^{-1} ;{ }^{1} \mathrm{H}$ NMR (400 MHz, $\left.\mathrm{CDCl}_{3}\right) \delta 2.37(\mathrm{~s}, 3 \mathrm{H}), 2.68(\mathrm{~d}, J=13.0 \mathrm{~Hz}, 1 \mathrm{H}), 2.88(\mathrm{~d}, J=13.0 \mathrm{~Hz}, 1 \mathrm{H}), 3.24(\mathrm{bs}, 1 \mathrm{H})$, $6.27(\mathrm{~s}, 1 \mathrm{H}), 7.01-7.04(\mathrm{~m}, 2 \mathrm{H}), 7.01-7.04(\mathrm{~m}, 2 \mathrm{H}), 7.20-7.22(\mathrm{~m}, 3 \mathrm{H}), 7.29(\mathrm{~d}, J=8.2$ $\mathrm{Hz}, 2 \mathrm{H}), 7.42-7.46(\mathrm{~m}, 3 \mathrm{H}), 7.69-7.74(\mathrm{~m}, 2 \mathrm{H}), 7.81(\mathrm{~d}, J=8.2 \mathrm{~Hz}, 2 \mathrm{H}), 7.88(\mathrm{~s}, 1 \mathrm{H}) ;{ }^{13} \mathrm{C}$ NMR $\left(100 \mathrm{MHz}, \mathrm{CDCl}_{3}\right) \delta 21.7,44.7,72.6,125.5,127.6,128.4,128.6,129.1,129.5$,

${ }^{10}$ Zhang, F. Y.; Corey, E. J. Org. Lett. 2000, 2, 1097-1100. 
129.9, 130.2, 130.9, 133.3, 133.5, 138.7, 142.2, 143.5, 144.3, 147.7; LRMS (APCI): Mass calcd for $\mathrm{C}_{25} \mathrm{H}_{23} \mathrm{O}_{3} \mathrm{~S}\left(\mathrm{M}+\mathrm{H}^{+}\right)$403.1. Found 403.1.Characterization data for 14: IR (neat) $1707,1610,1596,1319,1304,1147,1084 \mathrm{~cm}^{-1} ;{ }^{1} \mathrm{H}$ NMR $\left(400 \mathrm{MHz}, \mathrm{CDCl}_{3}\right) \delta$ $2.41(\mathrm{~s}, 3 \mathrm{H}), 3.81(\mathrm{~s}, 2 \mathrm{H}), 5.04(\mathrm{~s}, 1 \mathrm{H}), 5.52(\mathrm{~s}, 1 \mathrm{H}), 7.27-7.34(\mathrm{~m}, 11 \mathrm{H}), 7.42(\mathrm{t}, J=7.2$ $\mathrm{Hz}, 1 \mathrm{H}), 7.71(\mathrm{~d}, J=8.3 \mathrm{~Hz}, 2 \mathrm{H}), 7.85(\mathrm{~s}, 1 \mathrm{H}) ;{ }^{13} \mathrm{C} \mathrm{NMR}\left(100 \mathrm{MHz}, \mathrm{CDCl}_{3}\right) \delta 21.9$, $29.9,117.7,126.2,127.9,128.5,128.8,129.4,130.0,130.1,131.4,131.8,136.6,139.9$, 140.0, 140.1, 142.3, 145.1, 199.2; LRMS (APCI): Mass calcd for $\mathrm{C}_{25} \mathrm{H}_{23} \mathrm{O}_{3} \mathrm{~S}\left(\mathrm{M}+\mathrm{H}^{+}\right)$ 403.1. Found 403.1.

c) Base-mediated transformation of $\mathbf{1 3}$ to $\mathbf{1 2}$.

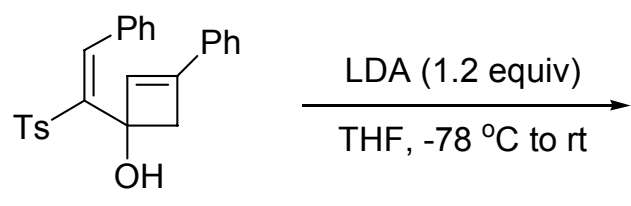

13<smiles>O=C1C=C(c2ccccc2)CC(=O)C1S(=O)(=O)OCl</smiles>

$12,94 \%$

To a solution of $12 \mu \mathrm{L}(0.086 \mathrm{mmol})$ of $i-\mathrm{Pr}_{2} \mathrm{NH}$ in $1 \mathrm{~mL}$ of dry THF, cooled to $78^{\circ} \mathrm{C}$, was added $43 \mu \mathrm{L}(0.086 \mathrm{mmol})$ of a $2 \mathrm{M}$ solution of $n$-BuLi in cyclohexane. After $10 \mathrm{~min}$ at bath temperature, a solution of $29 \mathrm{mg}(0.072 \mathrm{mmol})$ of alcohol 13 in $0.5 \mathrm{~mL}$ of THF was added dropwise over a period of 1 minute. Then the cooling bath was removed and the reaction mixture was stirred for $1 \mathrm{~h}$, after which time TLC (silica gel, EtOAc:Hex, 1:3) indicated complete consumption of starting material. The reaction mixture was quenched with $1 \mathrm{~mL}$ of saturated aqueous $\mathrm{NH}_{4} \mathrm{Cl}$ and then partitioned between $5 \mathrm{~mL}$ of water and $30 \mathrm{~mL}$ of $\mathrm{CH}_{2} \mathrm{Cl}_{2}$. The organic phase was dried $\left(\mathrm{MgSO}_{4}\right)$ and concentrated in vacuo to provide a light yellow residue, which was flash chromatographed over $15 \mathrm{~g}$ of silica gel (EtOAc:Hex, 1:3) to afford $27 \mathrm{mg}$ (94\%) of 12.

d) Preparation of 15 .
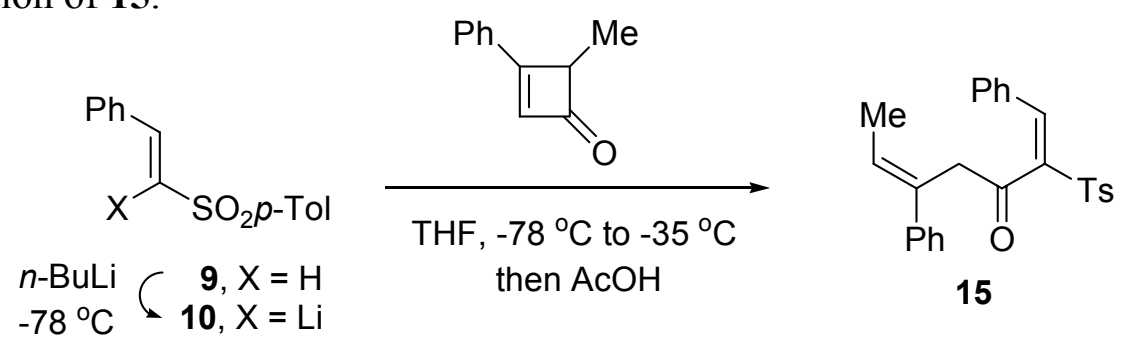

To a solution of $90 \mathrm{mg}(0.35 \mathrm{mmol})$ of sulfone $9 \mathrm{in} 1 \mathrm{~mL}$ of THF, cooled to -50 ${ }^{\circ} \mathrm{C}$, was added $0.18 \mathrm{~mL}(0.36 \mathrm{mmol})$ of a $2 \mathrm{M}$ solution of $n$-BuLi in cyclohexane dropwise. After 15 minutes at $-50{ }^{\circ} \mathrm{C}$, the reaction mixture was cooled to $-78{ }^{\circ} \mathrm{C}$. The red reaction mixture was then cannulated into a cooled $\left(-78{ }^{\circ} \mathrm{C}\right)$ solution of $50 \mathrm{mg}(0.32$ mmol) of 4-methyl-3-phenylcyclobutenone in $1 \mathrm{~mL}$ of THF. The reaction mixture was warmed to $-35^{\circ} \mathrm{C}$ and stirred for 1 hour at the bath temperature. After quenching with 50 $\mu \mathrm{L}$ of $\mathrm{AcOH}$ at $-35{ }^{\circ} \mathrm{C}$, the reaction mixture was warmed to room temperature and then concentrated in vacuo. The crude oil was flash chromatographed (hexanes:EtOAc, 5:1) to obtain $74 \mathrm{mg}$ of 1,5-diphenyl-2-(toluene-4-sulfonyl)hepta-1,5-dien-3-one (57\% yield) as a yellow oil. Characterization data: IR $\left(\mathrm{CDCl}_{3}\right) 3058,3027,2924,2858,1708,1597$, 
1146, 1318, 1146, $1085 \mathrm{~cm}^{-1}$; ${ }^{1} \mathrm{H}$ NMR $\left(400 \mathrm{MHz}, \mathrm{CDCl}_{3}\right) \delta 1.70(\mathrm{~d}, J=7.0 \mathrm{~Hz}, 3 \mathrm{H})$, 2.41 (s, 3H), 3.86 (s, 2H), 6.03 (q, $J=7.0 \mathrm{~Hz}, 1 \mathrm{H}), 7.14-7.18(\mathrm{~m}, 2 \mathrm{H}), 7.18-7.27(\mathrm{~m}, 5 \mathrm{H})$, 7.27-7.30 (m, 3H), 7.35-7.41 (m, 2H), $7.71(\mathrm{~d}, J=8.1 \mathrm{~Hz}, 2 \mathrm{H}), 7.84(\mathrm{~s}, 1 \mathrm{H}) ;{ }^{13} \mathrm{C} \mathrm{NMR}$ $\left(100 \mathrm{MHz}, \mathrm{CDCl}_{3}\right) \delta 14.5,21.7,46.0,126.3,126.7,128.1,128.4,128.6,129.1,129.7$, $129.8,131.0,131.6,131.8,136.4,139.7,142.1,142.4,144.8,198.3$; LRMS (APCI): Mass calcd for $\mathrm{C}_{26} \mathrm{H}_{25} \mathrm{O}_{3} \mathrm{~S}\left(\mathrm{M}+\mathrm{H}^{+}\right)$417.1. Found 417.1. 
$\mathrm{Ph}$

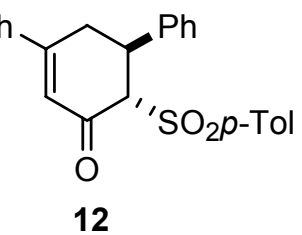

$\mathrm{CDCl}_{3}, 400 \mathrm{MHz}$

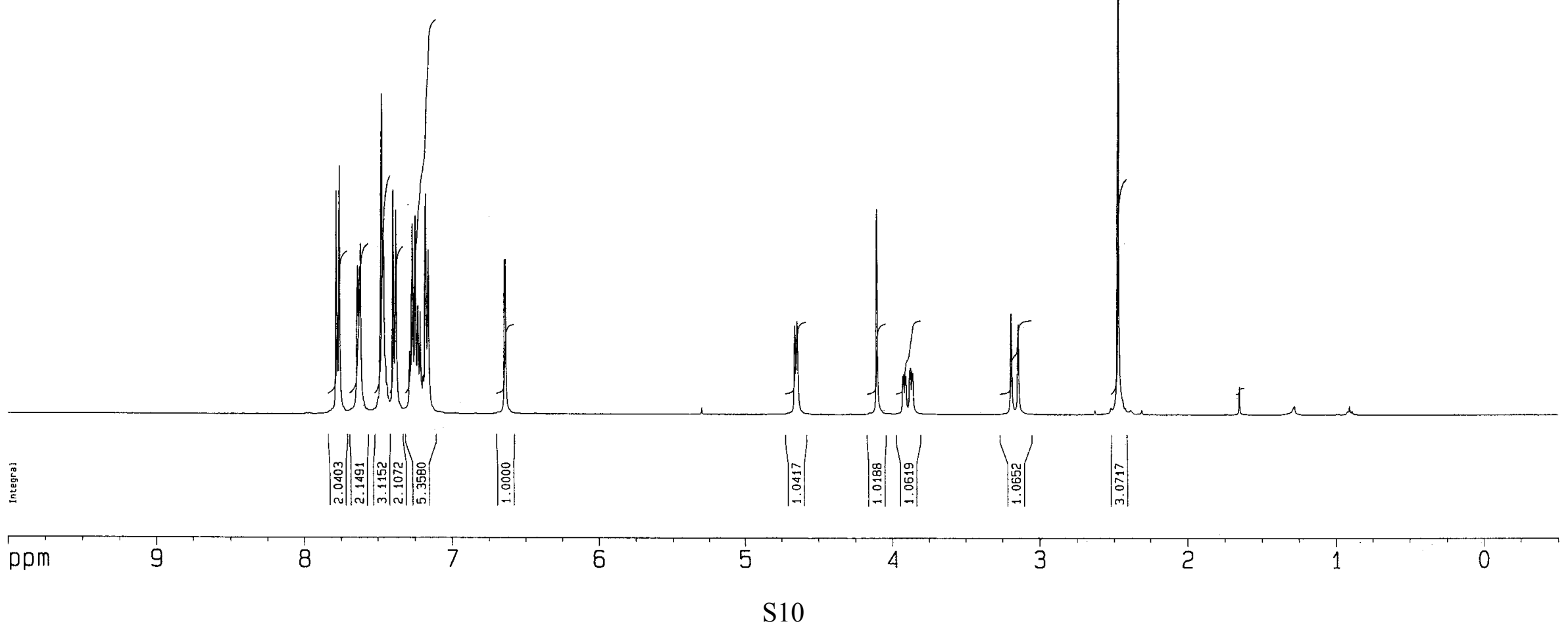




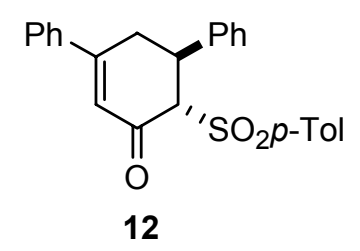

$\mathrm{CDCl}_{3}, 100 \mathrm{MHz}$

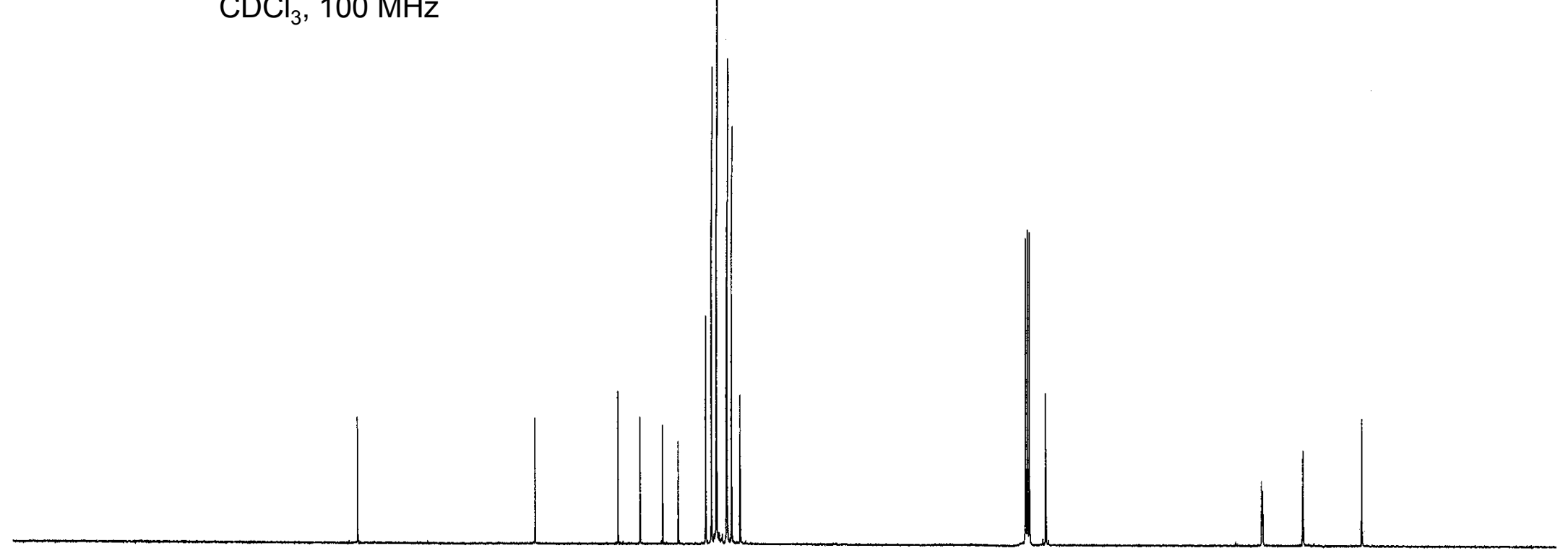

$\begin{array}{lllllll} & \\ p p m & 220 & 200 & 180 & 160 & 140 & 120\end{array}$

$120 \quad 100 \quad 80$

60

40

20 


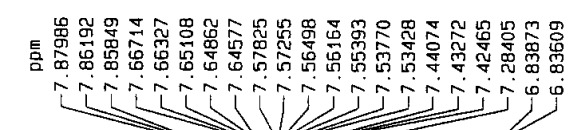

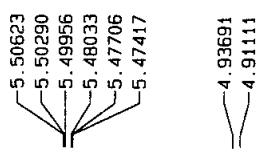

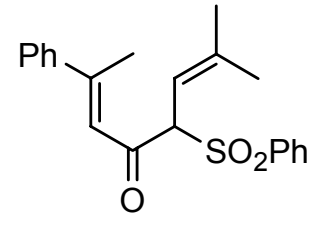

18

$\mathrm{CDCl}_{3}, 400 \mathrm{MHz}$

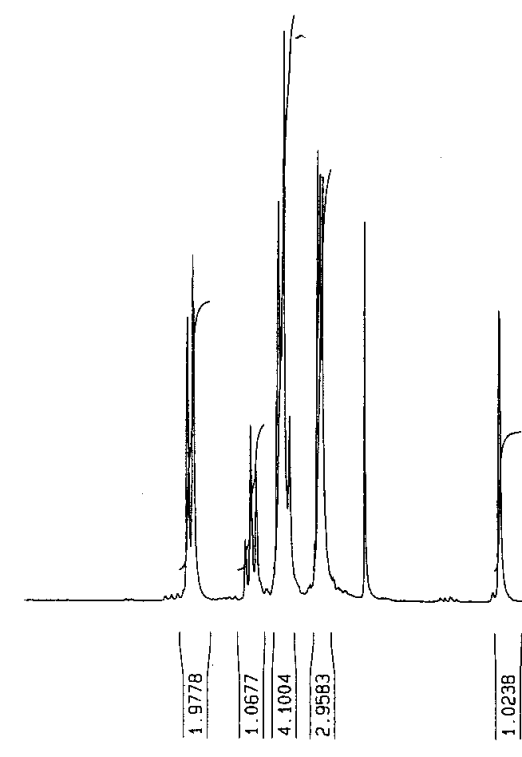

7.5

7.0

6.5

6.0

5.5

5.0

4.5

$4.0 \quad 3.5$

খ

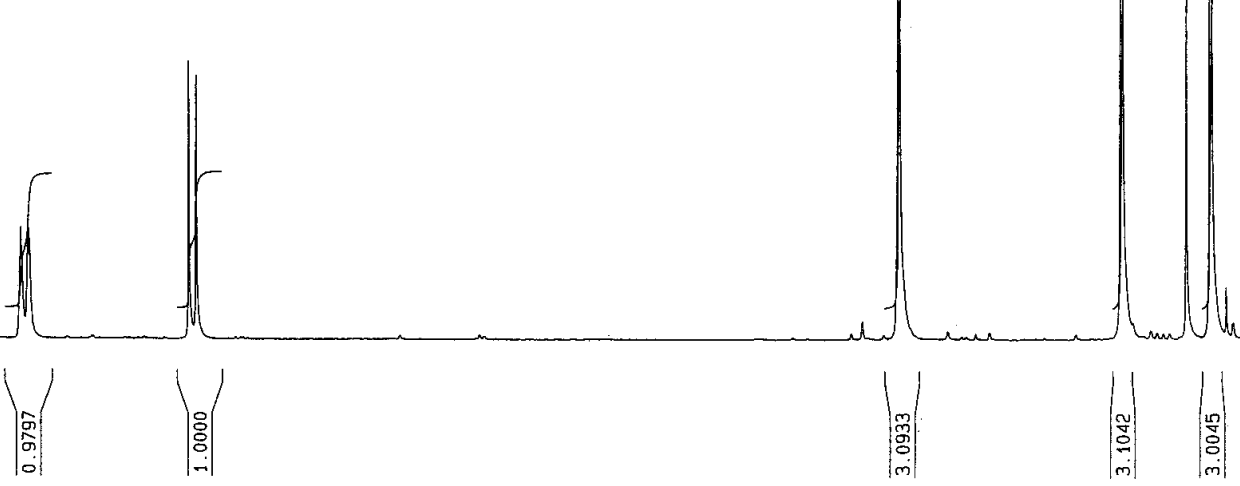



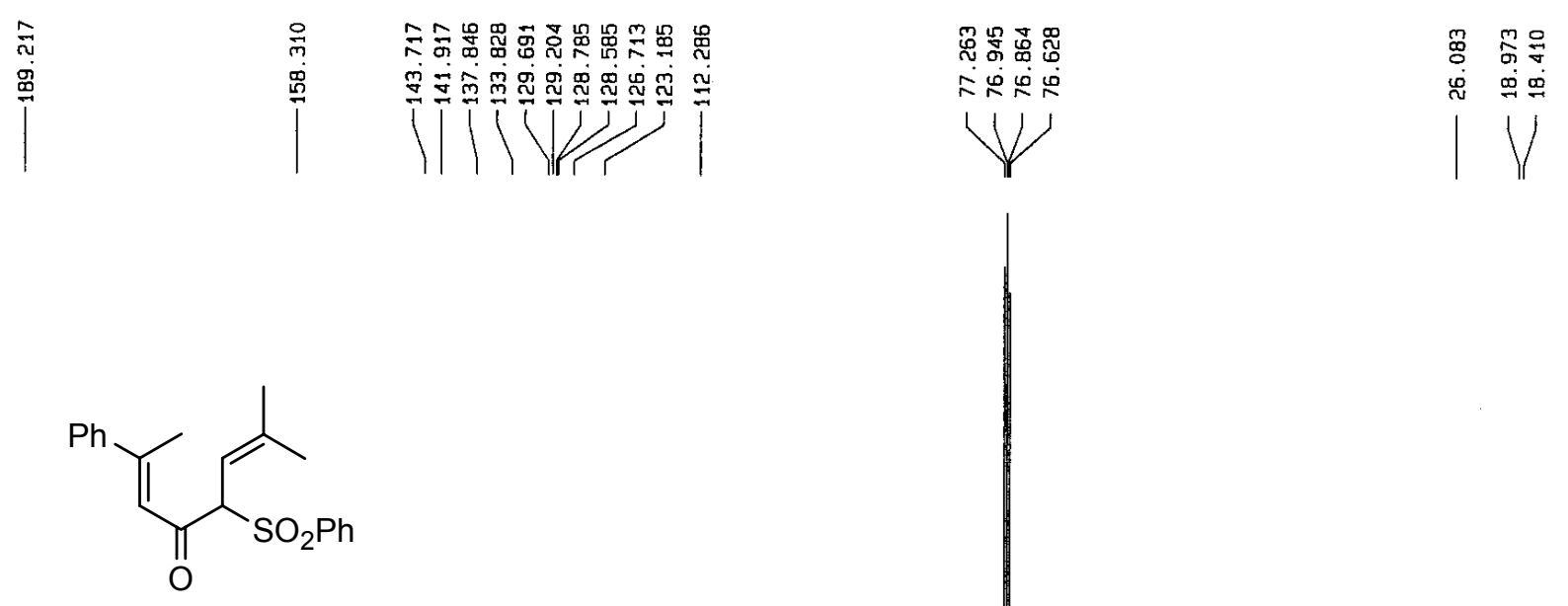

18

$\mathrm{CDCl}_{3}, 100 \mathrm{MHz}$

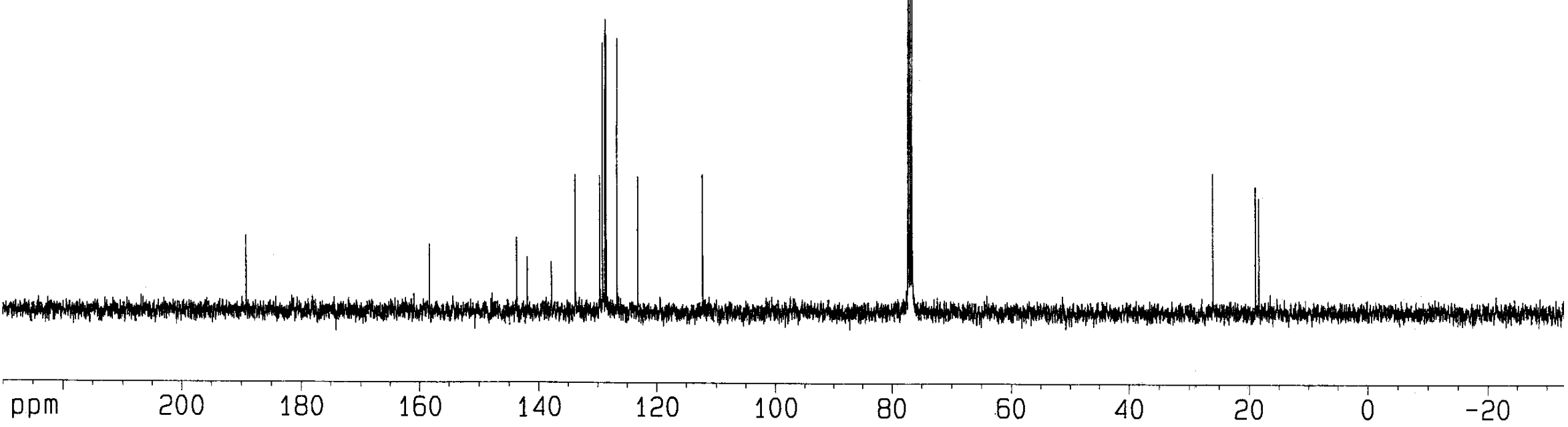


ฐ

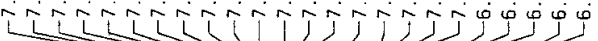

V

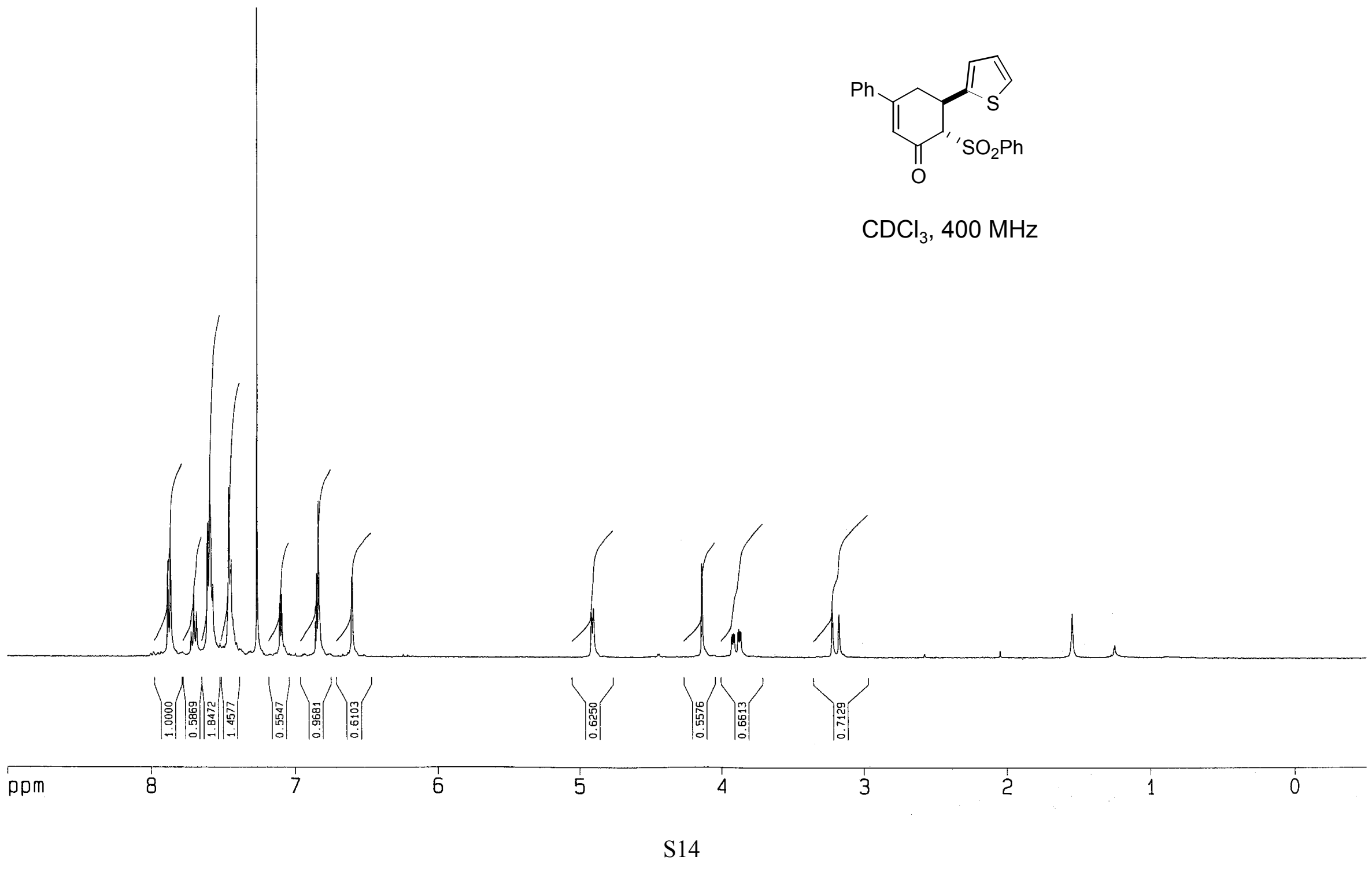




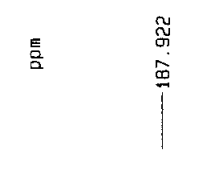

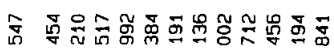

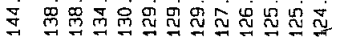

vivij

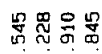

동

đ્م

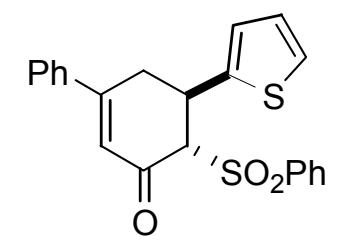

$\mathrm{CDCl}_{3}, 100 \mathrm{MHz}$

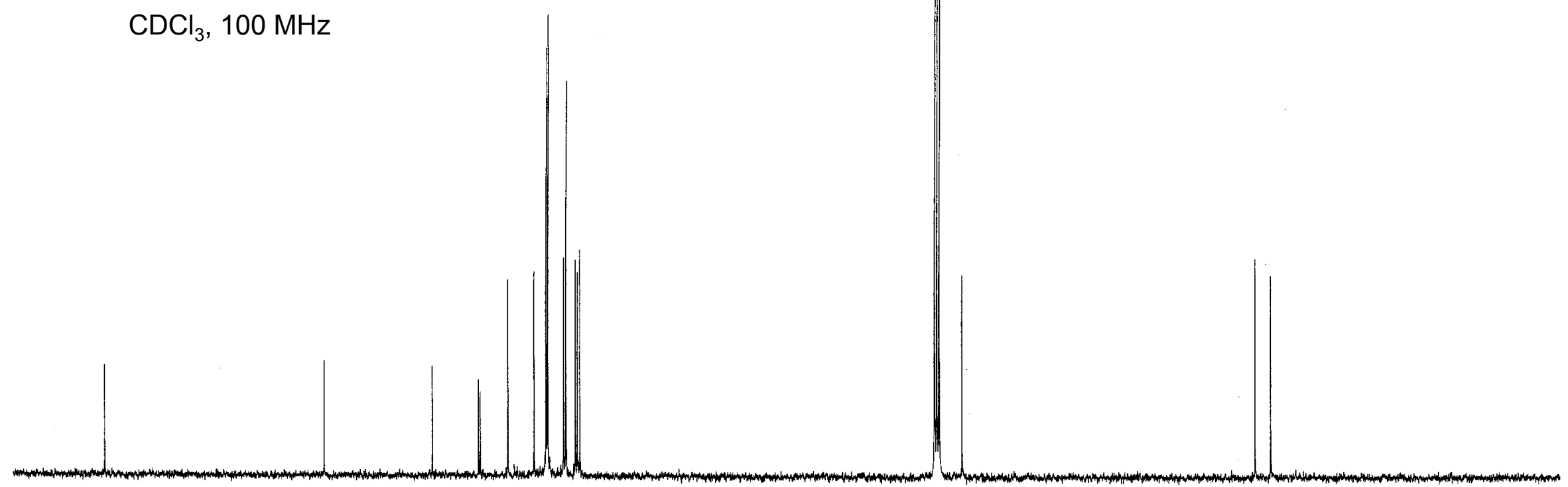


$\mathrm{Ph}$
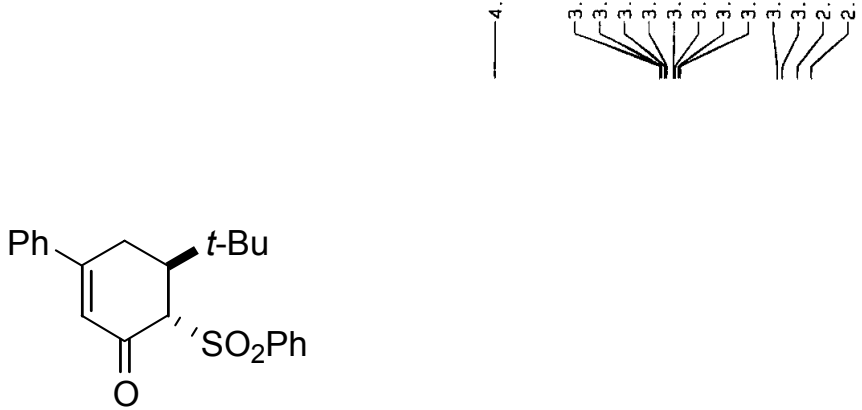

$\mathrm{CDCl}_{3}, 400 \mathrm{MHz}$

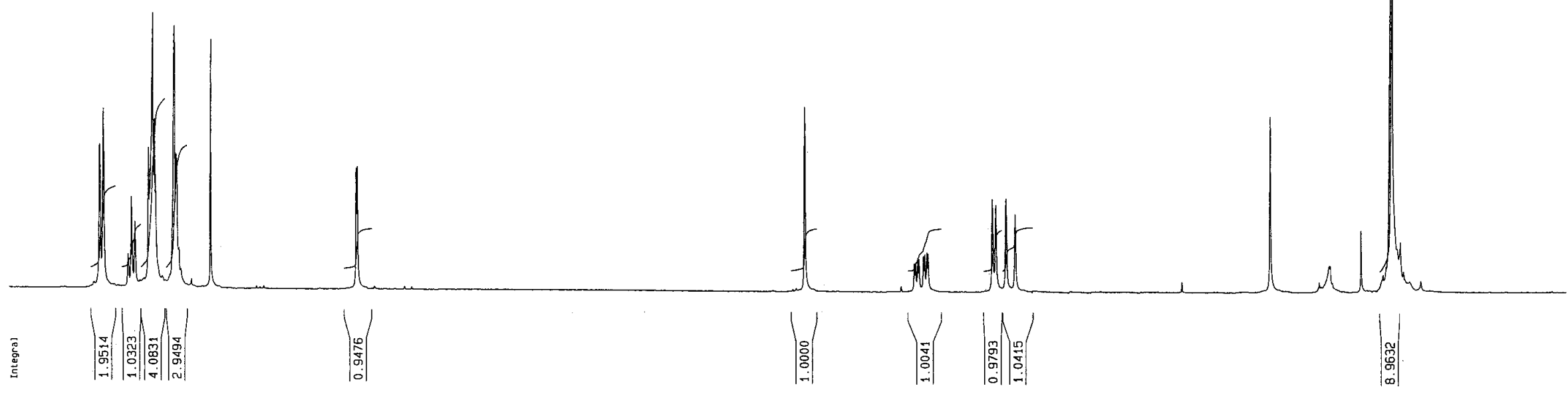

7.5

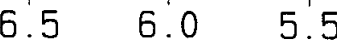

5.0

4.5

$4.0 \quad 3.5$

$3.0 \quad 2.5$

$\begin{array}{llll}2.0 & 1.5 & 1.0 & 0.5\end{array}$

S16 


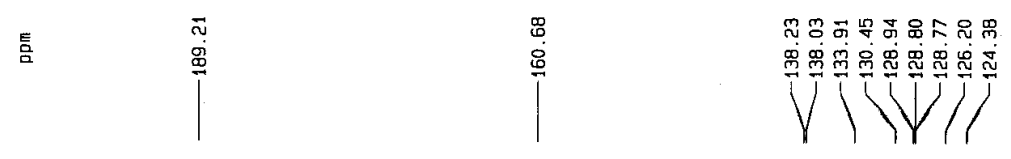

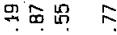

रिष्ट

V

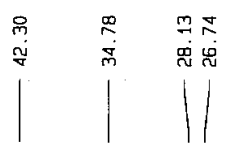<smiles>CC(C)(C)[C@H]1CC(c2ccccc2)=CC(=O)[C@H]1[S+](=O)(Oc1ccccc1)Oc1ccccc1</smiles>

$\mathrm{CDCl}_{3}, 100 \mathrm{MHz}$

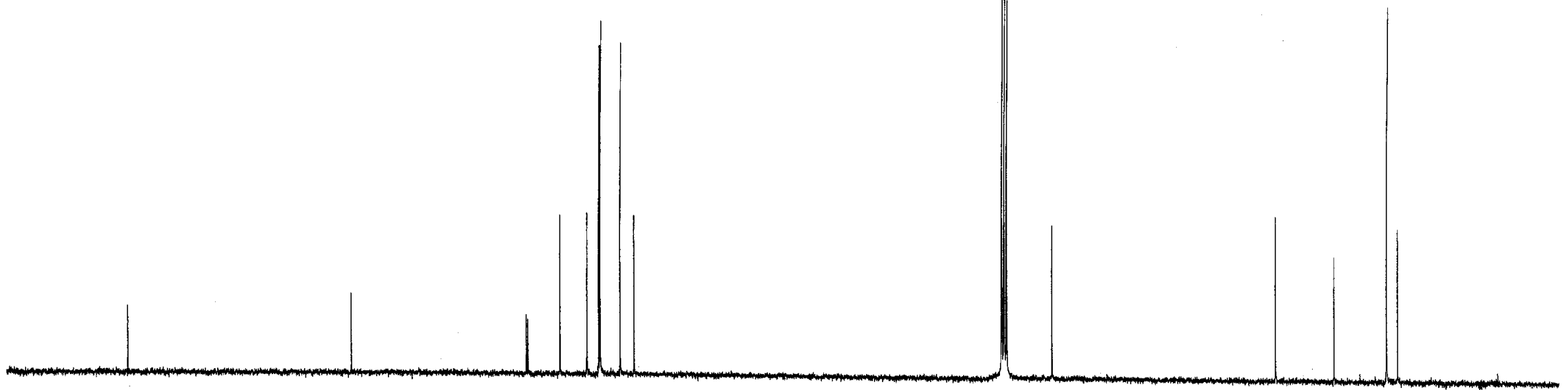




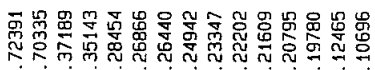

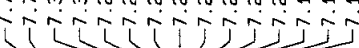

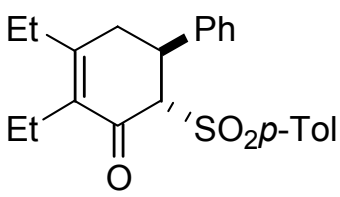

$\mathrm{CDCl}_{3}, 400 \mathrm{MHz}$
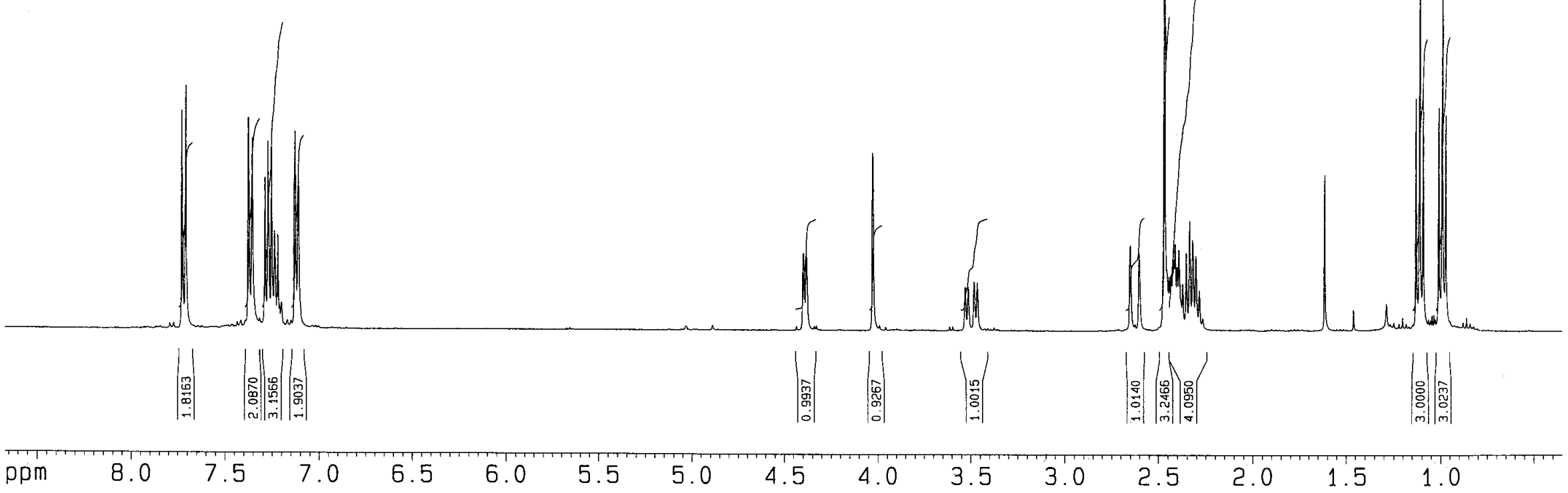

5.5

$5.0 \quad 4.5$

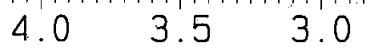

2.5

2. $0 \quad 1.5$

1. 0 


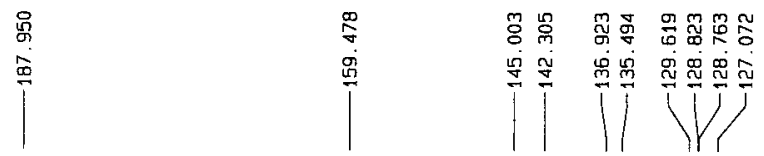

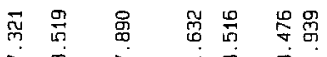

के

Et

$\prod_{\mathrm{O}}^{\mathrm{Ph}} \mathrm{SO}_{2} p$-Tol

$\mathrm{CDCl}_{3}, 100 \mathrm{MHz}$

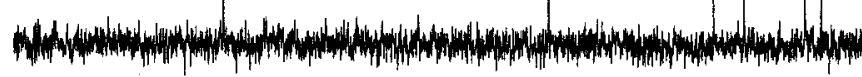




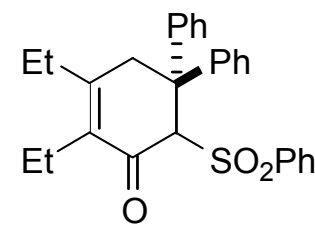

$\mathrm{CDCl}_{3}, 400 \mathrm{MHz}$
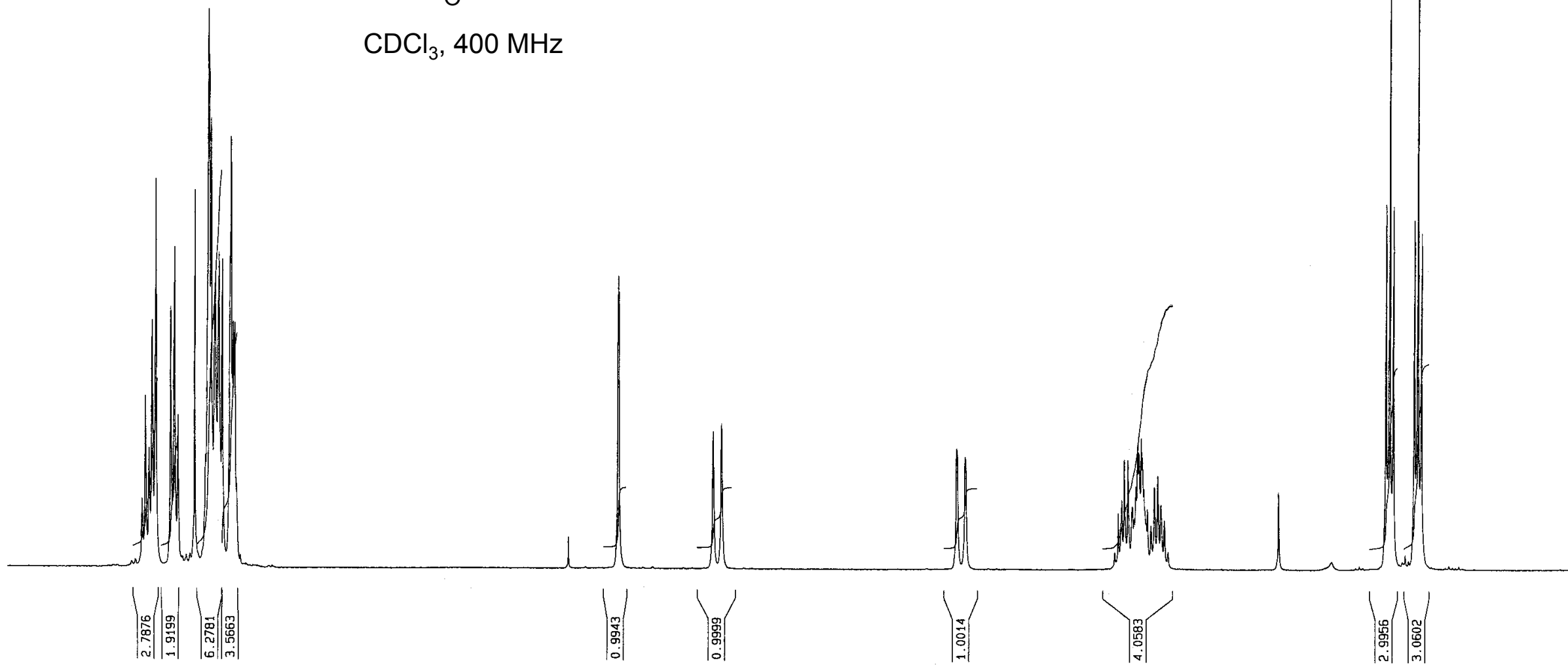


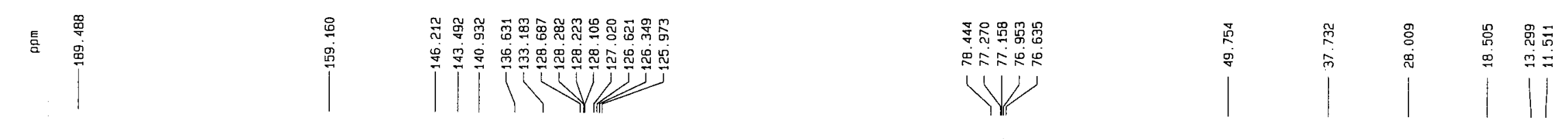

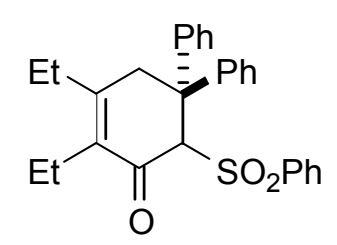

$\mathrm{CDCl}_{3}, 100 \mathrm{MHz}$

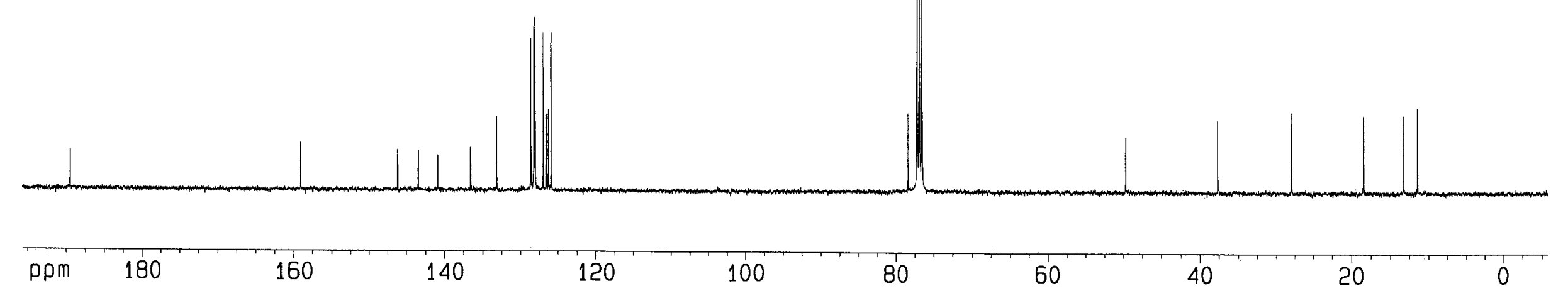




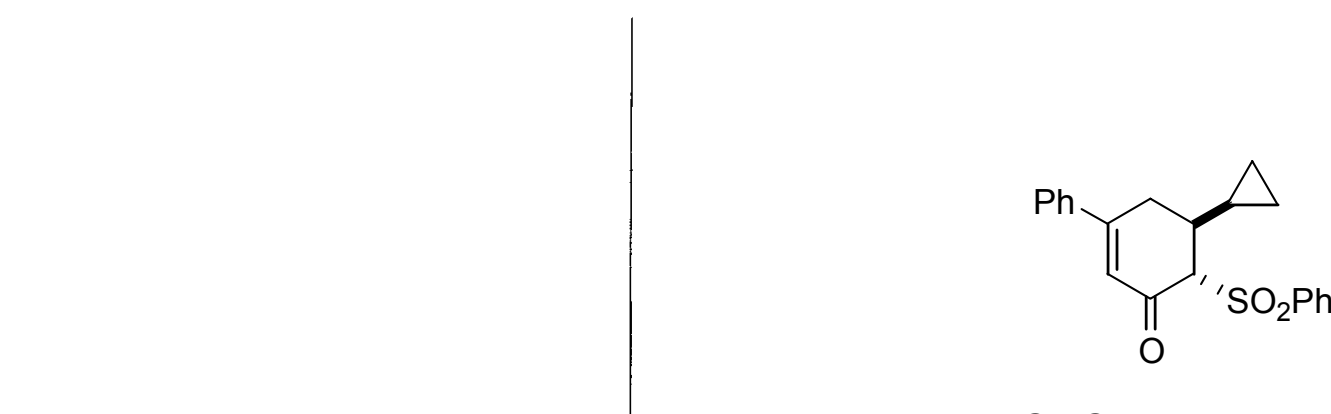

$\mathrm{CDCl}_{3}, 400 \mathrm{MHz}$

94:6 trans:cis in equilibrium

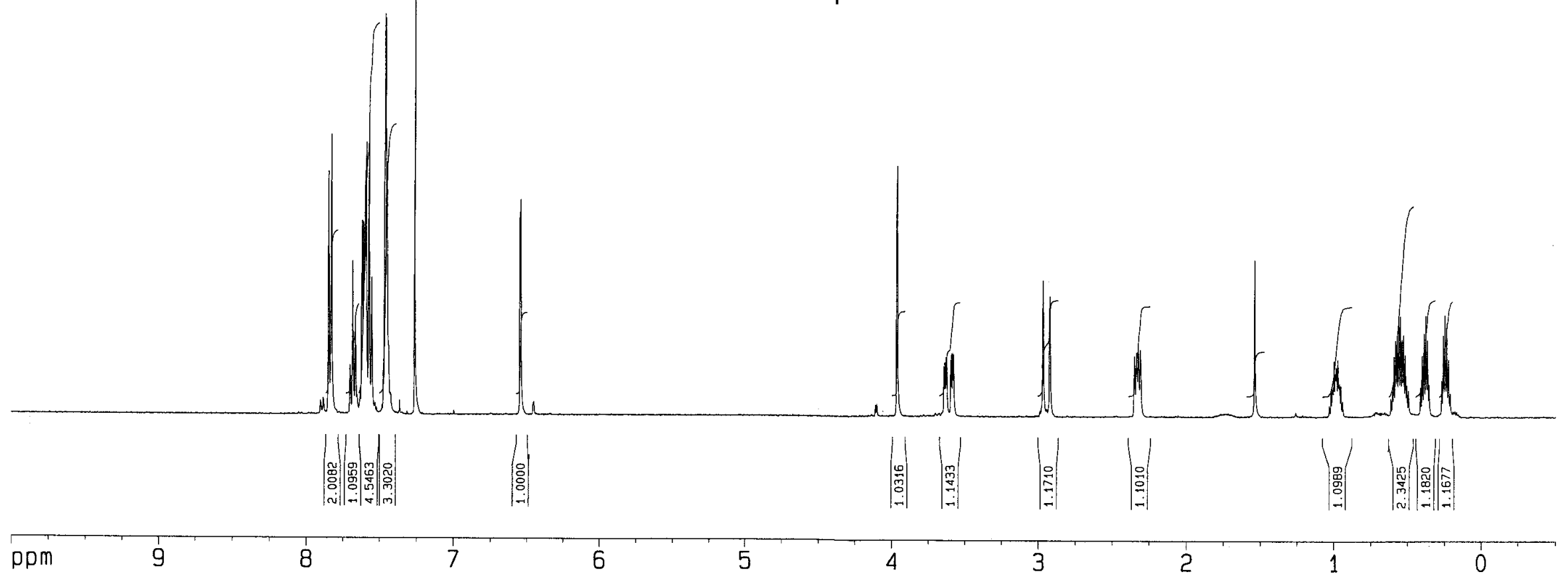




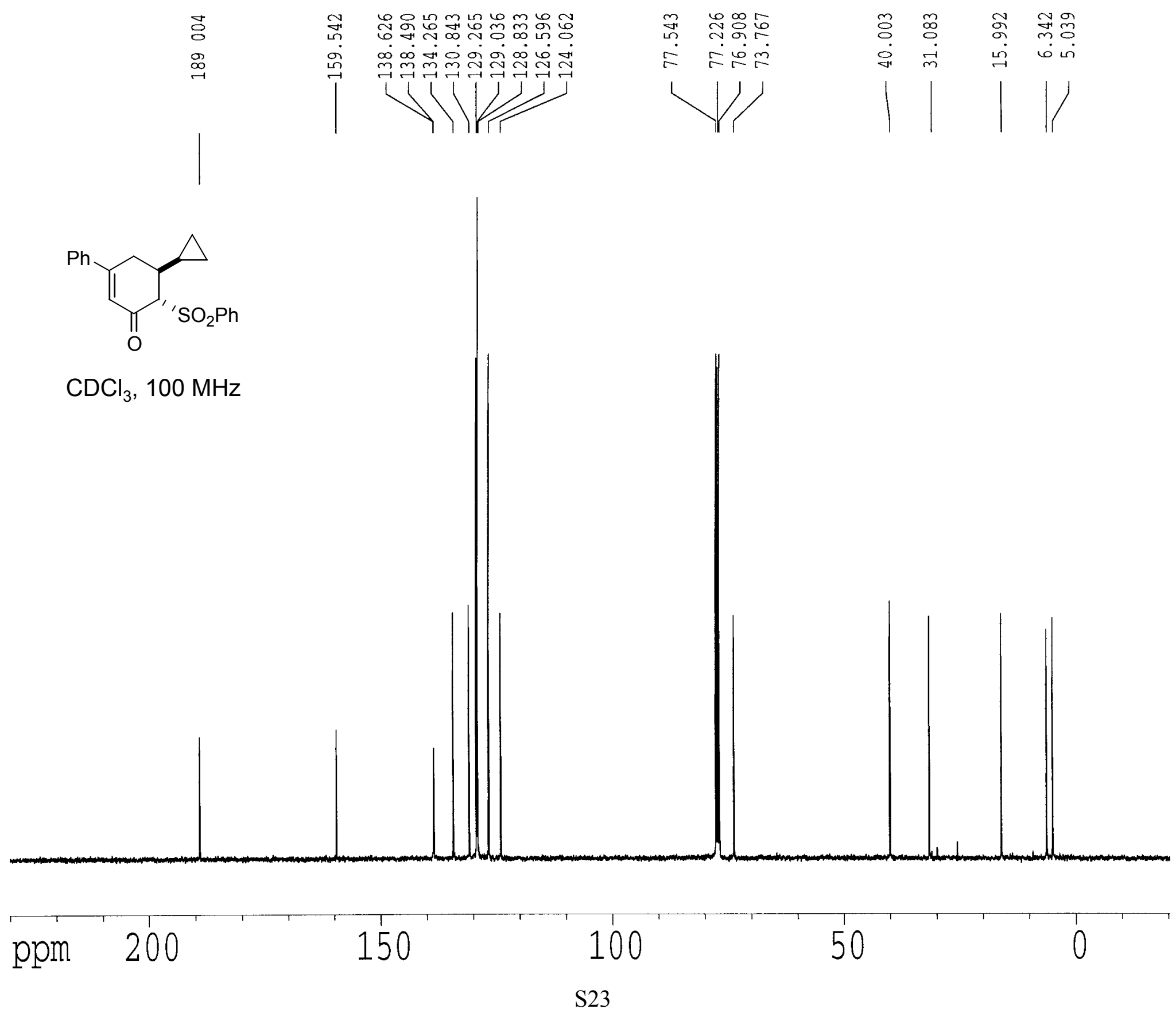




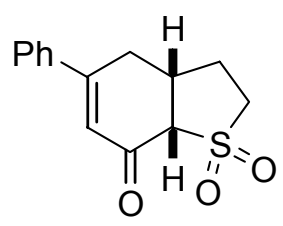

$\mathrm{CDCl}_{3}, 400 \mathrm{MHz}$

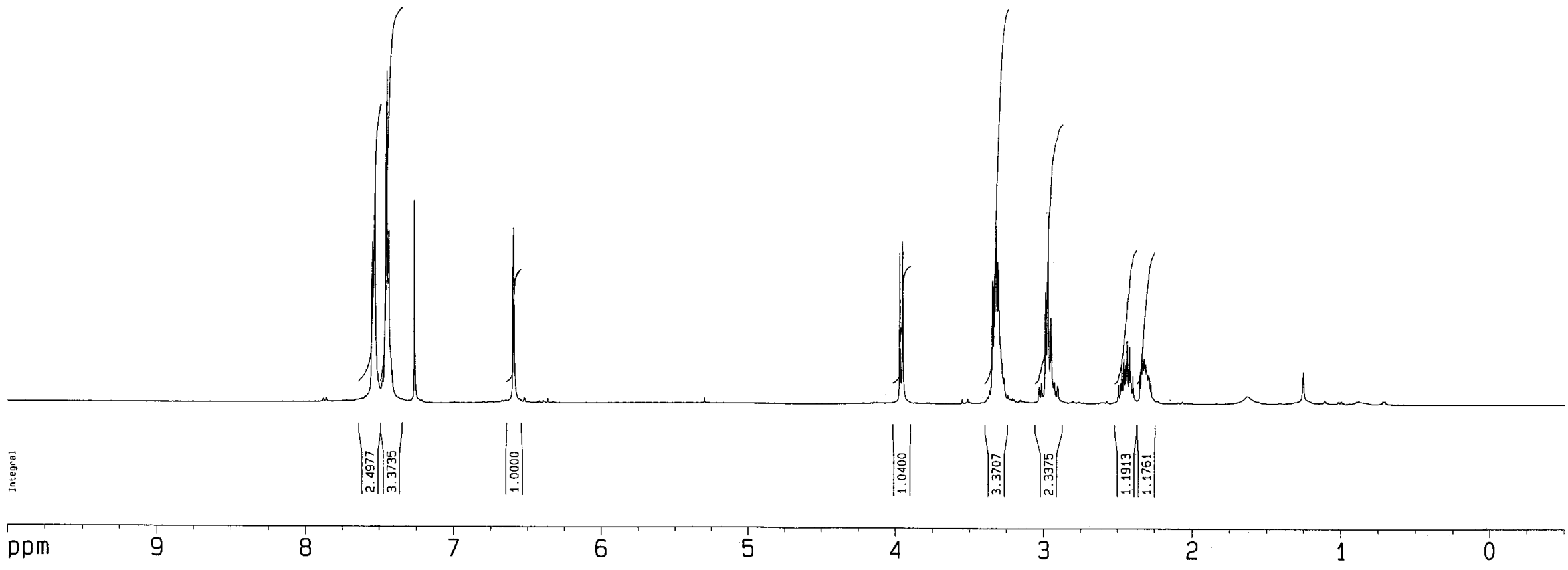




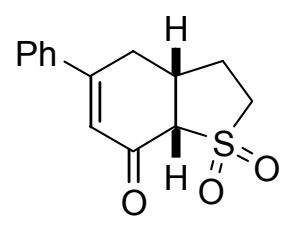

$\mathrm{CDCl}_{3}, 400 \mathrm{MHz}$

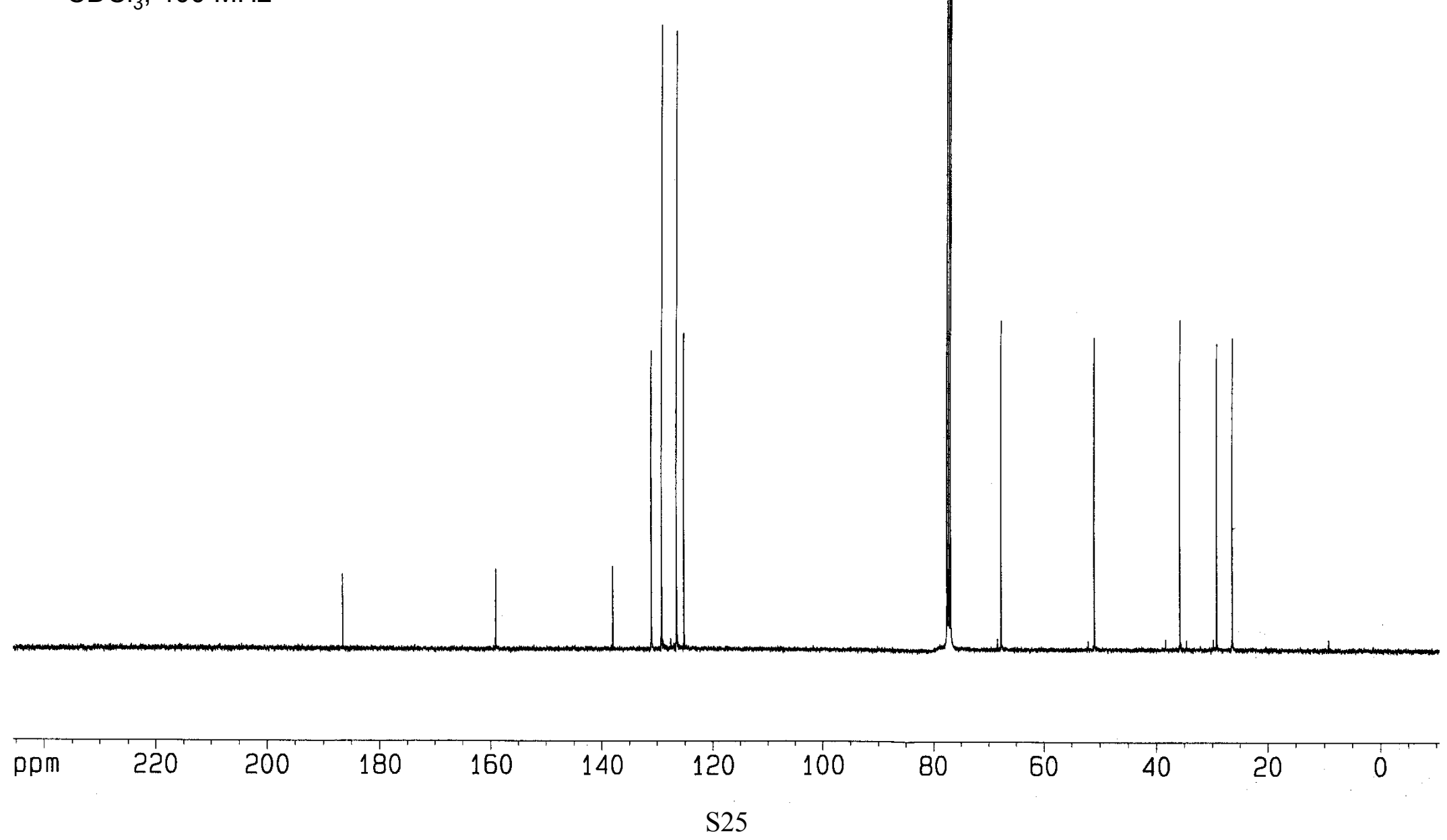



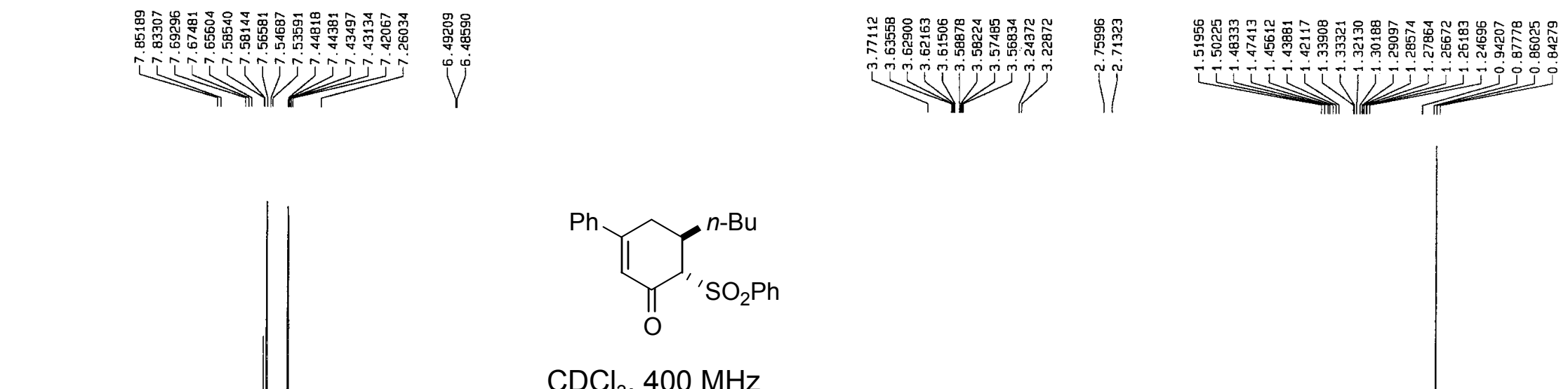

85:15 trans:cis in equilibrium
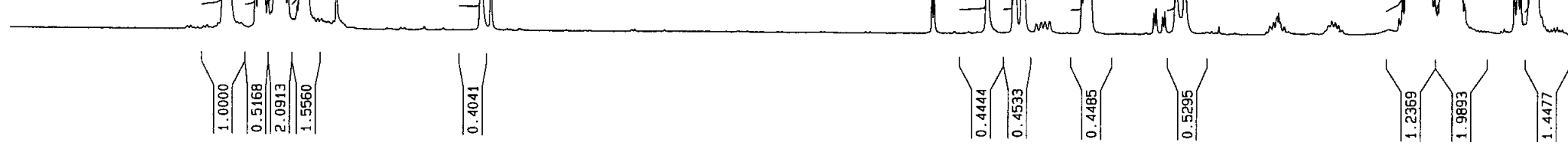

8

6

5

4

3

2

0 


辰

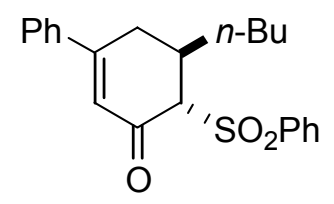

$\mathrm{CDCl}_{3}, 100 \mathrm{MHz}$

85:15 trans:cis in equilibrium

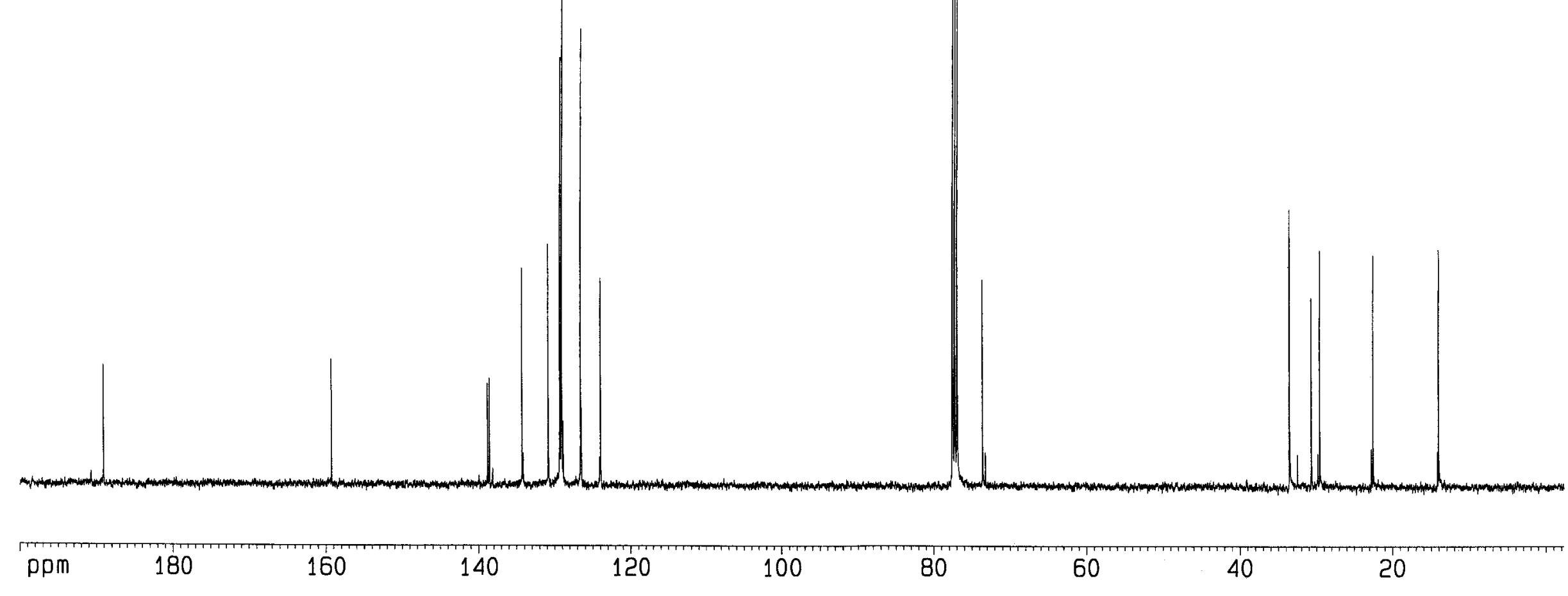



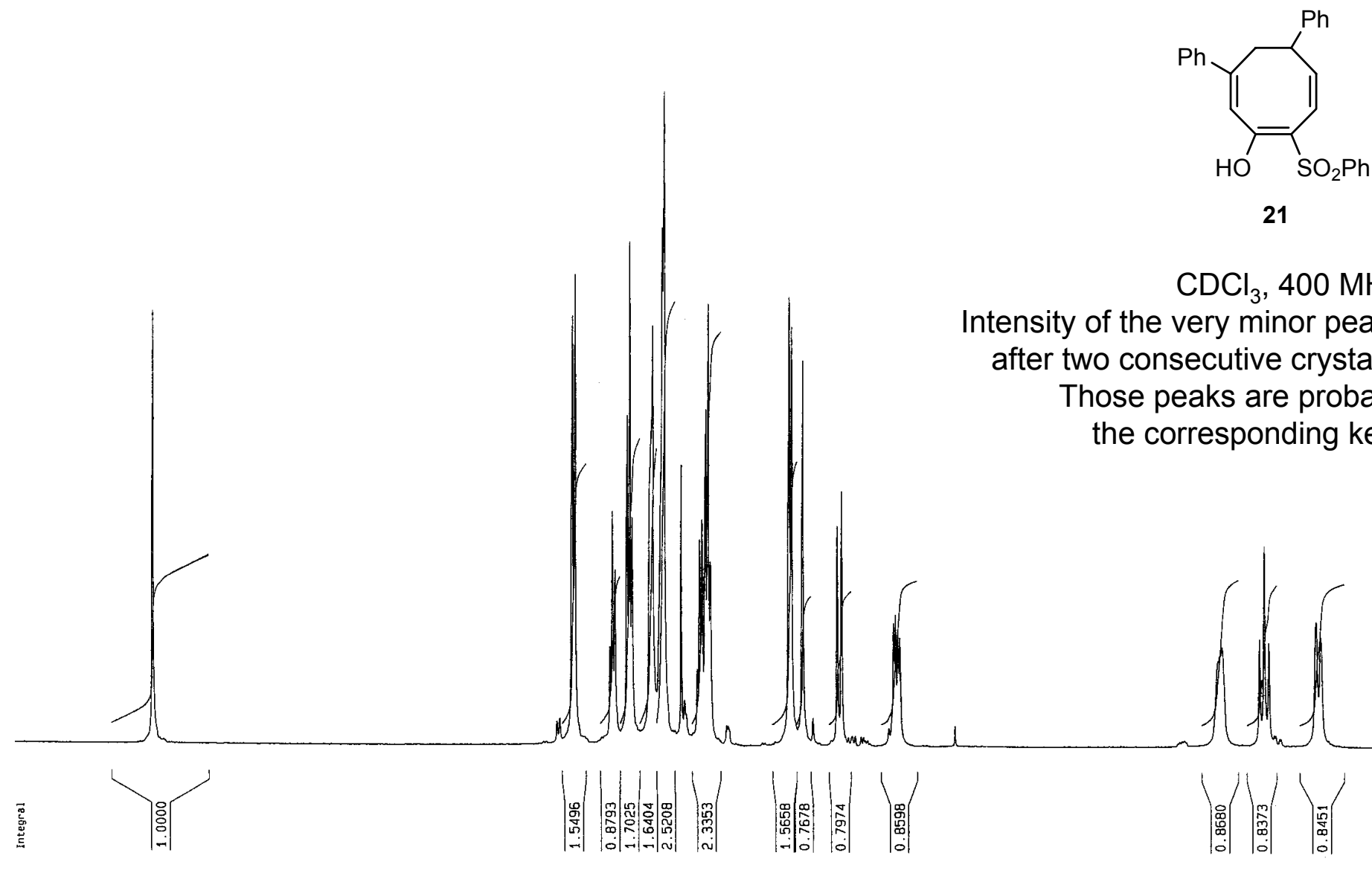

$\mathrm{CDCl}_{3}, 400 \mathrm{MHz}$

Intensity of the very minor peaks did not change after two consecutive crystallizations of $\mathbf{2 1 .}$

Those peaks are probably due to the corresponding keto form.

10

9

8

7

6

5

4

3

2

1 


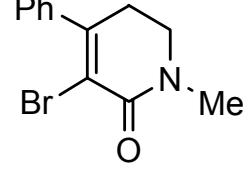

22

$\mathrm{CDCl}_{3}, 400 \mathrm{MHz}$

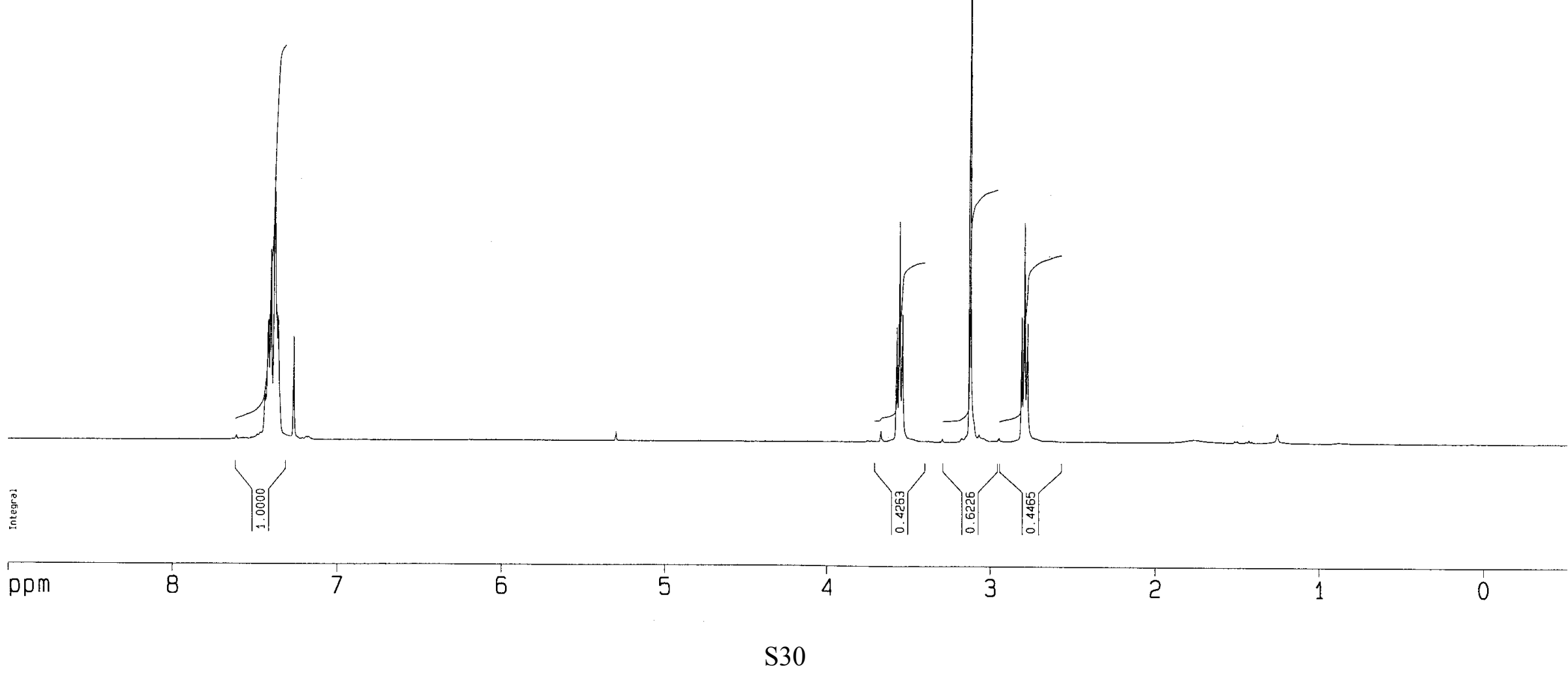




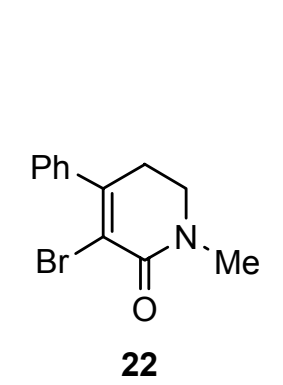

$\mathrm{CDCl}_{3}, 100 \mathrm{MHz}$ 

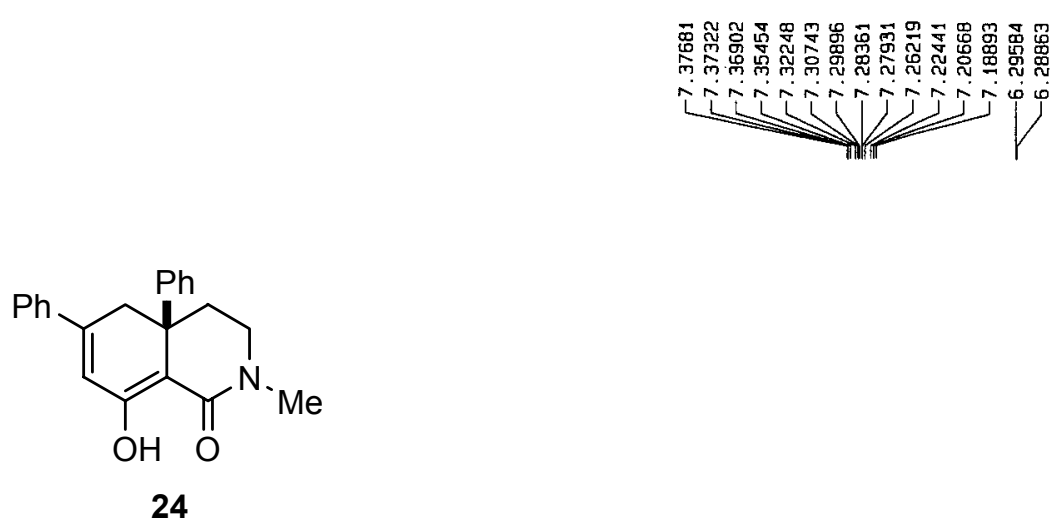

$\mathrm{CDCl}_{3}, 400 \mathrm{MHz}$

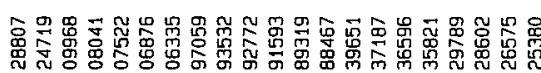

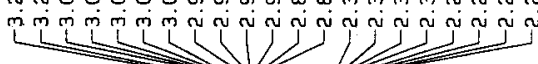

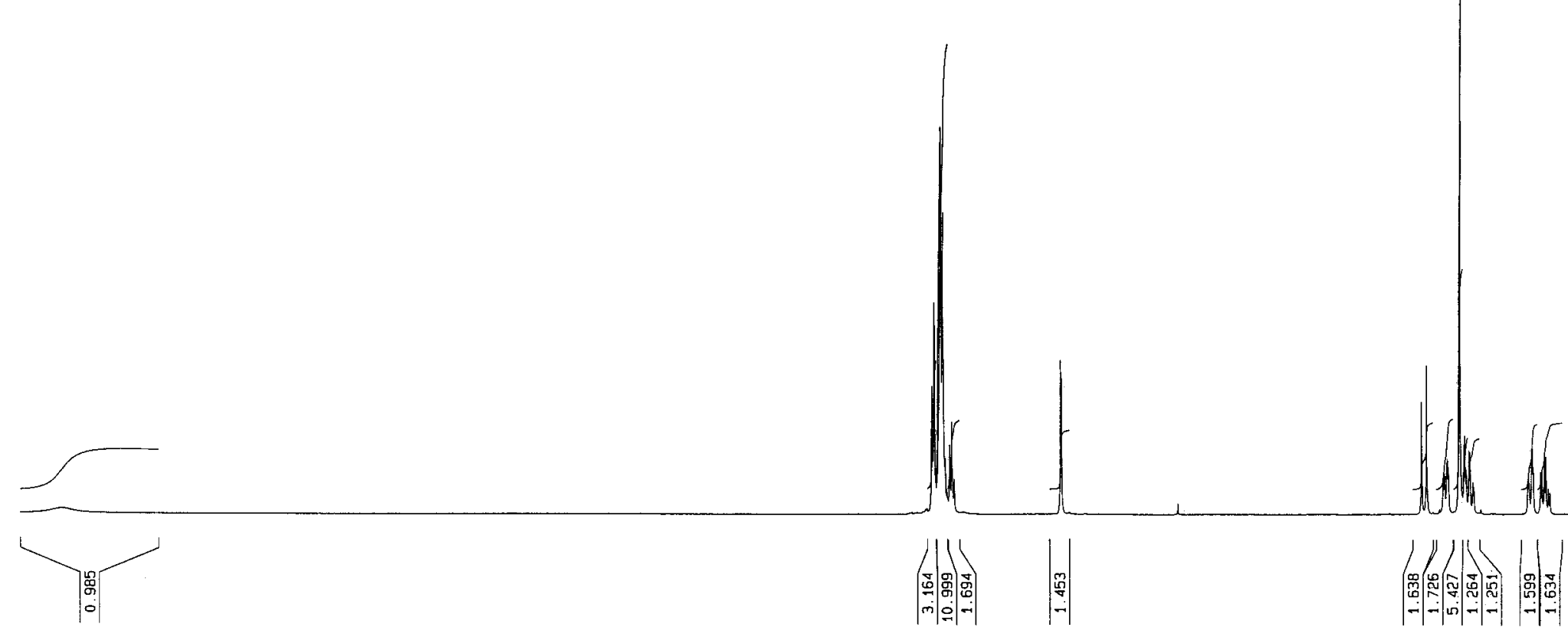

$$
p p m
$$

13

12

11

10

9

8

5

4

3

2 


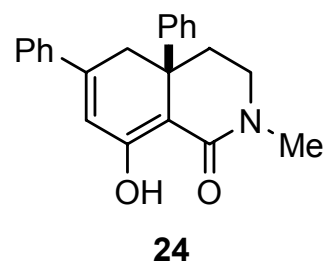

$\mathrm{CDCl}_{3}, 100 \mathrm{MHz}$ 